Int. J. Adhesion and Adhesives, vol 28, 2008, 222-236

\title{
Numerical analysis of the energy contributions in peel tests: a steady-state multilevel finite element approach
}

\author{
Ph. Martiny ${ }^{\mathrm{a}, *}$, F. Lani ${ }^{\mathrm{a}}$, A.J. Kinloch ${ }^{\mathrm{b}}$, T. Pardoen ${ }^{\mathrm{c}}$ \\ ${ }^{\mathrm{a}}$ CENAERO Centre de Recherche en Aéronautique, \\ Avenue Jean Mermoz 30, B-6041 Gosselies, Belgium \\ ${ }^{\mathrm{b}}$ Imperial College London, Mechanical Engineering Department, \\ Exhibition Road, London SW7 2AZ, UK \\ 'Université catholique de Louvain, Département des Sciences des Matériaux et des Procédés, \\ Unité d'Ingénierie des Matériaux et des Procédés, \\ Place Sainte Barbe 1, B-1348 Louvain-la-Neuve, Belgium
}

\begin{abstract}
The present work considers the numerical simulation of the steady-state fracture of adhesively bonded joints in various peel test configurations. The model is based on a multiscale approach involving the simulation of the continuum elastoplastic response of the adherends and the adhesive layer, as well as of the fracture process taking place inside the adhesive layer using a cohesive zone formulation. The model parameters are firstly identified by comparison with experimental results obtained with the wedge-peel test. Secondly, the ability of the model to predict peel test results obtained with different peel test configurations (e.g. wedge-peel or fixed-arm peel test geometries and various adhesive layer or arm thicknesses) is critically assessed by comparison with experimental data. Thirdly, the results of the steady-state simulations are post-processed in order to (i) evaluate the adhesive fracture energy, (ii) quantify and discuss the different contributions to plastic dissipation within the adhesive layer, and (iii) explain how these mechanisms affect the adhesive fracture energy as a function of the peel test configuration. The values of adhesive fracture energy, $G_{a}$, deduced from the numerical simulations proposed in the present paper, from all the various elastic-plastic peel test configurations, lie in the range of about $900 \pm 50 \mathrm{~J} / \mathrm{m}^{2}$; whilst the values from a previous analytical model and a node-release finite-element analysis model, for a cohesive fracture of the present adhesive, all lie in the range of about $1100 \pm 250 \mathrm{~J} / \mathrm{m}^{2}$. Thus, there is very good agreement between the different modelling methods. These values are clearly also in good agreement with the corresponding value from the well-established LEFM TDCB method of $1140 \pm 170 \mathrm{~J} / \mathrm{m}^{2}$.
\end{abstract}

\footnotetext{
${ }^{*}$ Corresponding author. Tel.: +32 71 919361; fax: +32 71919330.

E-mail address: philippe.martiny@cenaero.be (Ph. Martiny).
} 
Keywords: peel, steady-state fracture, adhesive fracture energy, plastic dissipation, finite element model, cohesive zone models. 


\section{Introduction}

The peel test is a very common technique for assessing the strength of adhesively bonded joints. There exists a large variety of peel test configurations [1-3], which differ from each other in the way the peel test is actually undertaken and other geometric features. Most of them consist in measuring the force per unit width, $P / b$, which is referred to as the peel strength that is required to peel apart the two adherends, or substrates, that are initially bonded together. Figure 1 shows the different test geometries that will be specifically dealt with in the present paper.

A simple energy balance shows that the peel strength scales with the total energy spent in the test. It includes the stored-strain energy and plastic dissipation in the peel arm, in addition to the adhesive fracture energy. As a consequence, not only can the measured values of the peel strength be very different from one test configuration to another, but they may also depart significantly from the value of the adhesive fracture energy, $G_{a}$, as measured via a linear-elastic fracture-mechanics test, for example [4].

Much work has been done in the last decade to correct, either experimentally [5] or analytically [4, 67], the measured peel strength for these extrinsic energy contributions. These approaches have been applied successfully to various peel test configurations, and the results tend to show that the adhesive fracture energy, $G_{a}$, is independent of the test configuration [4,7].

To take a closer look at the adhesive fracture energy as a function of the specimen geometry, Pardoen and co-workers [8] developed a quasi-static steady-state finite element model of the wedge-peel test, assuming symmetric failure of the specimens. In their model, the local fracture process is simulated with a cohesive zone, where the local energy dissipation in the adhesive, ahead of the crack front, is accounted for by embedding this cohesive zone in between layers of elastic-plastic solid elements. The adhesive fracture energy derives naturally from this distinction and is evaluated as:

$$
G_{a}=\Gamma_{0}+\Gamma_{p}
$$

where $\Gamma_{0}$ is the intrinsic work of fracture associated with the cohesive zone and $\Gamma_{p}$ is the sum of the farfield stored-strain energy and the energy dissipation in the solid elements within the adhesive layer. Therefore, the value of $G_{a}$ does not include any contribution from the energy dissipation occurring in the adherends, such as plastic bending. The results obtained in [8] showed a dependence of $G_{a}$ upon both the adhesive layer and adherend thicknesses that was explained by the authors in terms of internal and external constraint effects. It is interesting to note that simpler approaches such as those of Yang et al [9] or Ferracin et al [10], which describe the entire adhesive layer with a single row of cohesive elements, do not separate $\Gamma_{0}$ and $\Gamma_{p}$ and cannot therefore capture any possible dependence of $\Gamma_{p}$ on the detailed geometry of the peel test. 
More recently, Martiny et al [11] has extended the research initiated by Pardoen and co-workers [8, 10] and made an attempt to account for the dependence upon the arm thickness by subtracting from $G_{a}$ the energy contributions to $\Gamma_{p}$ that are not directly caused by the local fracture process. The present paper describes a further advance to this approach with the aims of (i) extending the model to other peel test configurations, (ii) critically assessing the former by comparison with experimental results, and (iii) examining the effect of the test configuration on the local plastic dissipation in the adhesive and, hence, on the value of the adhesive fracture energy, $G_{a}$.

\section{Experimental}

This study focuses on a particular adhesive/adherend system. The adherend material was aluminum alloy (AA) 5754-O and is supplied in the form of $1.00 \mathrm{~mm}$ and $1.45 \mathrm{~mm}$ thick sheets. The adhesive was Permabond ESP110 (Bondmaster, Eastleigh, UK) which is a general purpose, rubber-toughened, epoxy-based paste adhesive. Experimental data were gathered for these materials for two purposes: calibrating the model and assessing the accuracy of the modelling predictions.

\subsection{Tensile tests}

Three dumbbell-shaped specimens were machined from both $1.00 \mathrm{~mm}$ and $1.45 \mathrm{~mm}$ thick aluminumalloy 5754-O sheets and tested in tension using a universal testing machine with a crosshead speed of 1 $\mathrm{mm} / \mathrm{min}$. The corresponding stress versus strain curves are shown in Figure 2. Small differences can be observed as a function of the thickness of the aluminum-alloy specimens; and these differences have been taken into account in the present modelling studies. The identification of the hardening law parameters from these data will be presented in Section 3.5 below.

Similarly, eight round tension-test specimens were machined from a single bulk adhesive plate, which was molded following the recommendations given in [12] and cured for 45 minutes at $150^{\circ} \mathrm{C}$. The specimens were tested using a universal testing machine with crosshead speeds ranging from 0.1 to 10 $\mathrm{mm} / \mathrm{min}$, in order to quantify any possible rate-dependent behavior. The corresponding curves are shown in Figure 3. From this plot, it can be seen that the adhesive is slightly rate-dependent. However, it was considered that this small effect could be safely neglected in the present studies. The identification of the flow-law parameters from these data will also be presented in Section 3.5.

\subsection{Wedge-peel tests}

Three distinctly different specimen geometries, but with identical thicknesses for the two arms, were tested using the wedge-peel test. They correspond to three different combinations of adhesive layer, $h_{a d h}$, and adherend thickness, $h$, respectively: (i) $h_{a d h}=0.25 \mathrm{~mm}$ and $h=1.00 \mathrm{~mm}$, (ii) $h_{a d h}=0.25 \mathrm{~mm}$ and $h=1.45 \mathrm{~mm}$, and (iii) $h_{a d h}=0.40 \mathrm{~mm}$ and $h=1.45 \mathrm{~mm}$.

The specimens were prepared from $200 \mathrm{~mm}$ x $20 \mathrm{~mm}$ aluminum-alloy strips cut from sheets of the appropriate thickness. The surfaces of the strips were prepared by grit blasting followed by a chromic acid etch. The adhesive thickness was controlled by embedding steel wires in the adhesive layer, either $0.25 \mathrm{~mm}$ or $0.40 \mathrm{~mm}$ in diameter, and the adhesive was then cured for 45 minutes at $150^{\circ} \mathrm{C}$. Three 
specimens were tested for each geometry employing the set-up schematically presented in Figure 1(a). Fracture was always cohesive in the adhesive layer, but near one adhesive/adherend interface. By convention, the side of the specimen close to that which the crack propagated will be called the 'crack side', whereas the side on which most of the adhesive remains will be termed as the 'adhesive side'. The wedge thickness was equal to $1.50 \mathrm{~mm}$ and was inserted in the adhesive layer at an advancing rate of $7.5 \mathrm{~mm} / \mathrm{min}$. After testing, the residual radii of curvature of the arms were measured, on both sides of the adhesive layer, as an indicator of the overall energy expended in the test. These radii will be denoted by $R_{1}$ and $R_{2}$ on the adhesive and on the crack side, respectively, and were measured via photographic measurements. The crack length, $a$, defined as the axial distance between the crack tip and the contact points with the wedge, was also measured using an optical method. It should be noted that, since the force required to push the wedge is significantly affected by friction, the opening driving force is not a useful parameter for any subsequent analysis.

The experimental results pertaining to the wedge-peel test are gathered in Table 1 , and several noteworthy comments are to be made. The measured radii of curvature show a moderate to large variation from one specimen to the other for a given coupon geometry. Moreover, the larger the measured radius of curvature, then the larger the associated scatter; probably because the relative variation in the overall energy expenditure is smaller. Values of $R_{2}$ on the crack side are systematically smaller than on the adhesive side, $R_{1}$. As a reminder, the detached arm on the crack side is made of the metallic adherend only while, on the adhesive side, it is covered with the adhesive which increases its bending stiffness and explains the larger values of $R_{1}$. For an increasing adhesive layer thickness, the value of $R_{1}$ increases while both $a$ and $R_{2}$ decrease. Assuming that the adhesive layer is fully plastic, an increase in $h_{a d h}$ makes the adhesive/adherend sandwich (i.e. on the adhesive side) more compliant at crack tip, in the direction normal to the crack plane. Therefore, a shorter crack length and, hence, a larger bending moment are needed to produce sufficient crack opening. This increased bending moment causes the value of $R_{2}$ on the crack side to drop while $R_{1}$ still increases because the gain, associated to the larger value of $h_{a d h}$, in bending stiffness of the adhesive/adherend sandwich dominates. Finally, for an increasing adherend thickness, overall the flexural stiffness increases, so that the crack length can become longer to produce the same opening effect, and both radii of curvature increase.

\subsection{Fixed-arm peel tests}

Fixed-arm peel tests have been performed during the course of a previous study [13]. Therefore, they will be only briefly described in the present paper. A single joint geometry is considered and it was manufactured using a $1.00 \mathrm{~mm}$ thick AA 5754-O 'flexible' adherend bonded to a $10.0 \mathrm{~mm}$ thick AA 5754-O 'rigid' substrate with a $0.40 \mathrm{~mm}$ thick layer of ESP110 adhesive. Specimens $220 \mathrm{~mm}$ x $20 \mathrm{~mm}$ in dimensions were prepared following a procedure similar to the one described above for the wedgepeel test specimens. The aluminum-alloy adherends were grit blasted and chromic acid etched before application of the adhesive, which was cured for 45 minutes at $150^{\circ} \mathrm{C}$. The desired adhesive layer thickness was obtained by placing steel wires, with the appropriate diameter, within the adhesive layer prior to bonding. 
The fixed-arm peel test specimens were bolted through their base plate onto the experimental set-up shown in Figure 1(b) and tested using a crosshead speed of $5 \mathrm{~mm} / \mathrm{min}$ at three different peel angles: 45, 90 and 135 degrees. They all showed a failure which was cohesive in the adhesive layer, but near the interface of the adhesive/flexible adherend. The peel force versus time trace was recorded and an average, steady-state peel force per unit width, $P / b$, was deduced. The maximum curvature of the peel arm, $1 / R_{0}$, was also numerically derived from digital photographs taken during the course of the test.

The fixed-arm peel test results are given in Table 2. They show that the maximum curvature of the flexible peeling arm increases with the peel angle, since the plastic bending becomes more intense. Nevertheless, the peel force decreases at the same time as the lever (i.e. peel) arm available for producing the bending moment increases.

\section{The model}

\subsection{General description}

The present model is an extension to the AACZ model introduced by Pardoen et al [8], where AACZ

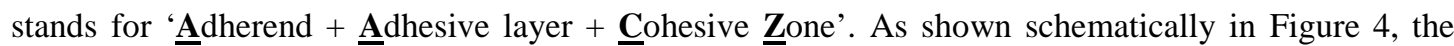
model relies on the assumption that the damage mechanisms (e.g. bond breakage, void nucleation, growth and coalescence) leading to fracture are localized in a thin layer of material ahead of the crack tip called the 'fracture process zone'. Applying the model initially proposed by Tvergaard and Hutchinson [14] on interfacial debonding to cohesive (near-the-interface) failures, it describes this local fracture process with the help of a traction versus separation law across the crack surface and further accounts for the inelastic deformations around the crack by a rate-independent isotropic $J_{2}$ theory of plasticity for the adherends and the adhesive.

The particular traction versus separation law that is used is also shown in Figure 4(b). It relates the normal traction, $\sigma$, to the normal opening displacement, $\delta$, across the crack surface and shows no resistance in shear, since fracture is assumed to be predominantly via a Mode I (tensile) failure. It is characterized by the peak stress, $\hat{\sigma}$, that can be sustained and the critical opening displacement, $\delta_{c}$, above which the normal tractions drop to zero. The area under the curve is denoted by $\Gamma_{0}$ and represents the intrinsic work of fracture, i.e. the energy dissipated per unit area of crack advance in the fracture process zone by the local damage mechanisms leading to fracture. It can therefore also be viewed as the value of the adhesive fracture energy, $G_{a}$, at crack initiation.

The implementation of the model relies on a 2D plane-strain, large rotation, quasi-static steady-state finite element formulation, which is well suited to modelling peel tests. Indeed, typical dimensions for the specimens make the stress state mostly 2D plane-strain across the width of the adhesive bond. The deformed geometry exhibits large displacements, particularly at the tip of the arms but at the test speeds employed in the present work there are no significant dynamic effects. Finally, the steady-state deformed geometry of the specimen is obtained in a single calculation; whilst transient models, such as those of Cui et al [15], require the computation of numerous transient steps before reaching steady-state 
conditions.

Considering the details of the present model, the displacement field is approximated, in the finite element sense, across the structure and solved using the weak form of the principle of virtual work. As a convenient way to account for large rotations, this latter is expressed in terms of Green-Lagrange strains $E_{i j}$ and second order Piola-Kirchhoff stresses $S_{i j}$ :

$$
\int_{\Omega_{0}} S_{i j} \delta E_{i j} d \Omega_{0}=\int_{\Omega_{0}} f_{i} \delta u_{i} d \Omega_{0}+\int_{\Sigma_{0}} t_{i} \delta u_{i} d \Sigma_{0}
$$

where integration is performed on the initial, i.e. undeformed, volume $\Omega_{0}$. In the above equation, $\delta E_{i j}$ is obtained analytically from the expression of the finite element approximation to the displacement field via the definition of Green-Lagrange strains. The isotropic $J_{2}$ flow theory is used to relate stress increments to strain increments, with the accumulated plastic strain $p$ as the only internal variable:

$$
\dot{S}_{i j}=\dot{S}_{i j}\left(\dot{E}_{k l}, p\right)
$$

In the steady-state formalism, the material velocity, $\mathbf{V}$, is imposed in the initial geometry. Throughout this paper, it will be taken, by convention, to be equal to:

$$
\mathbf{V}=(-1,0,0)
$$

Material is considered to flow in the negative $O X$ direction and the longitudinal axis of the specimen must therefore be aligned with that same direction. As a consequence, all material derivatives can be expressed in terms of spatial derivatives with respect to the material coordinate $X$ in the initial configuration:

$$
\dot{(\cdot)}=\mathbf{V} \cdot \frac{\partial(\cdot)}{\partial \mathbf{X}}
$$

and, in particular, using Equation (4):

$$
\dot{E}_{i j}=-\frac{\partial E_{i j}}{\partial X}
$$

so that the stress rate is fully determined by Equation (3). Stresses can then be evaluated by integrating numerically the latter along streamlines using a backward Euler scheme. This is more conveniently done in the initial geometry, where streamlines, considering the choice made in Equation (4), correspond to lines with constant $Y$ value: 


$$
S_{i j}^{(2)}=S_{i j}^{(1)}-\int_{X_{1}}^{X_{2}} \dot{S}_{i j}\left(-\frac{\partial E_{k l}}{\partial X}, p\right) d X .
$$

This completes the set of equations that is solved iteratively by a procedure that was first suggested by Dean and Hutchinson [16], see also [17], and applied to the present model by Pardoen et al [8].

\subsection{Wedge-peel test}

The wedge-peel test is modelled within the above framework by defining the geometry and associated boundary conditions. These are depicted in Figure 5 . Well ahead of the crack tip, the specimen is not affected by the loading and remains undeformed. Far behind the wedge, it is completely unloaded and shows a uniform curvature. As a consequence, the model can be restricted to some distances, $l_{u}$ and $l_{d}$, respectively ahead of the crack tip and past the wedge without any loss of information. This necessitates the application of appropriate boundary conditions to substitute for the missing portions. The inlet section (see Figure 5) is clamped in order to impose zero deformation and to fix rigid body modes at the same time. The outlet section (see Figure 5) should ideally be constrained so as to give a zero material derivative of the curvature. However, such conditions are difficult to formulate and to implement. It is much easier to extend the arms by a length $l_{d, e x t}$ past the outlet section and let the resulting end section be free. If $l_{d, e x t}$ is sufficiently large, then the outlet section is unaffected by the presence of the free end and behaves as if the arms were extending to infinity. In practice, lengths $l_{d}$ and $l_{d, \text { ext }}$ are merged into a single value $l_{d, t o t}$ that is increased until a significant portion of the arms past the wedge shows a uniform curvature. The outlet section can then equally be placed in this portion. Similarly, the length $l_{u}$ is increased until the computed crack tip opening displacement remains unchanged for a given crack length and wedge thickness. The choice of these lengths is part of the convergence study which is run for all analyses.

It was mentioned in Section 2 that the tests conducted in the present work all showed loci of failure that were cohesive in the adhesive layer, but which were near to an adhesive/adherend interface. Hence, for the sake of simplicity, the cohesive elements are placed at the adhesive/adherend interface. This is equivalent to neglecting both the compliance of, and the plastic dissipation occurring in, the thin layer of adhesive located between the crack and the interface. Moreover, the extent of these cohesive elements is restricted to the region ahead of the crack tip, i.e. they span the length $l_{u}$. This greatly reduces the need for a large number of iterations in the solution procedure, that would be otherwise required to break the cohesive elements past the crack tip. The disadvantage of this approach is that the crack length, $a$, is unknown a priori; and its value must be found by iteration until the condition is satisfied that the opening displacement at the assumed crack tip is indeed equal to its critical value $\delta_{c}$.

The presence of the wedge is taken into account by imposing the following multi-point constraint:

$$
v_{B}-v_{A}=D_{w}
$$

where vertical displacements $v_{A}$ and $v_{B}$ are taken, as an approximation, on the adhesive/adherend 
interfaces to avoid local straining in the adhesive. It should be noted that the axial component of the force imposed by the wedge, as well as the possible misalignment of points A and B in the deformed geometry, are also neglected. All of these simplifications are valid providing the thickness of the wedge, $D_{w}$, is small compared with the crack length, $a$.

\subsection{Fixed-arm peel test}

The geometry and boundary conditions used to simulate the fixed-arm peel test conditions are shown in Figure 6, and explained below. Since the rigid adherend is much thicker than the flexible adherend, and tightly fastened to the experimental set-up, it can reasonably be considered as being infinitely rigid. Therefore, it does not need to be modelled and can be substituted for by imposing zero displacements on the rigid adhesive/adherend interface. (Preliminary calculations confirmed that this assumption was reasonable.) Well ahead of the crack tip, the specimen is unaffected by the loading. Under steady-state conditions, the peel arm can be considered, to a first approximation, as being straight and parallel to the direction of the applied force over a finite length located somewhere in between the crack tip and the extremity of the peel arm. As the test proceeds further, this portion extends steadily towards the grips of the testing machine. Consequently, the model can be restricted to some distances, $l_{u}$ and $l_{d}$, respectively ahead of, and past, the crack tip. To account for the segments that are discarded, the inlet section is clamped and the outlet section is tied to an external length of the arm, $l_{d, e x t}$; which is introduced in the steady-state formulation and to which the external load is applied. To be rigorous, this length of the peel arm should be the true segment of the arm which makes the connection between the grips of the test machine and the zone of zero curvature, which consists of the initially unbonded segment of the peel arm supplemented with the length that was debonded before reaching steady-state conditions. However, since the complex distribution of plastic strains it encloses is unknown, it was replaced by the approximation of an elastic material free of any residual plastic strains. In practice, lengths $l_{d}$ and $l_{d, e x t}$ are merged in a single value $l_{d, t o t}$ that is chosen to be sufficiently long to obtain converged results. The boundary of the domain of interest is then interrogated in this range so as to provide an average material velocity aligned with the direction of the applied force. The procedure for determining the length $l_{u}$ is similar to that employed in the model of the wedge-peel test. Also, as for the model of the wedge-peel test, the cohesive elements are introduced at the adhesive/adherend interface over a length $l_{u}$.

\subsection{The mesh}

The models of the specimens were meshed using eight-noded quadrilateral elements. These elements are aligned with the reference coordinate system $O X Y$, just as the specimen in its undeformed geometry (see Figures 5 and 6), to form a structured mesh. This choice was made to facilitate numerical integration along streamlines, since Gauss points are aligned with iso- $Y$ lines in this arrangement.

For a given geometry and associated boundary conditions, the accuracy of the finite element solution is ensured by increasing the mesh refinement until convergence is attained. This requirement is monitored in terms of the work expended in each layer of material and the opening displacement at the crack tip. Accurate solutions are achieved with a minimum number of elements when using smaller mesh sizes in 
the vicinity of the crack tip and larger mesh sizes in the arms, while at the same time keeping the aspect ratio of the elements as close as possible to unity to avoid ill-conditioning. For the sake of computational efficiency, these guidelines were followed throughout the study. Two examples of such meshes are shown in Figure 7.

\subsection{Identification of the material parameters}

For the above model to be representative of the adhesive/adherend system under consideration, suitable values for the material parameters must be identified. These parameters characterize the bulk behaviour of both the adhesive and adherend materials, and the local fracture process within the adhesive. As noted above, the bulk behavior of the materials is modelled using isotropic $J_{2}$ theory of plasticity. The corresponding material parameters consist of the elastic properties (i.e. the Young's modulus, $E$, and Poisson's ratio, v), a mathematical expression for the hardening law and the associated coefficients. With the exception of Poisson's ratio, they are all derived by least-square fitting, in the small-strain range (i.e. $\varepsilon<3 \%$ ), the tensile stress versus strain curves presented in Section 2 . The aluminum-alloy, 5754-O, curves are fitted separately for each sheet material thickness, with the following equation which makes use of the empirical hardening law first suggested by Swift [18]:

$$
\sigma=\left\{\begin{array}{cl}
E \varepsilon & \left(\varepsilon \leq \sigma_{0} / E\right) \\
\sigma_{0}+h\left(\varepsilon-\frac{\sigma}{E}\right)^{n} & \left(\varepsilon>\sigma_{0} / E\right)
\end{array} .\right.
$$

The resulting fits are presented in Figure 2 and show good agreement with the experimental data, which supports the choice made for the expression for the hardening law. The tensile curves for the adhesive are fitted using the following equation:

$$
\sigma=\left\{\begin{array}{cc}
E \varepsilon & \left(\varepsilon \leq \sigma_{0} / E\right) \\
\sigma_{0}+h\left(\varepsilon-\frac{\sigma}{E}\right)^{n}+k\left(\varepsilon-\frac{\sigma}{E}\right) & \left(\varepsilon>\sigma_{0} / E\right)
\end{array}\right.
$$

Here, an additional term was added to Swift's hardening law in order to better match the experimental data, see Figure 3. The value of the Poisson's ratio could not be determined experimentally, since lateral contractions of the traction specimens were not measured in the tests. Therefore, it is taken to be equal to 0.40 and 0.33 for the adhesive and both aluminum alloys, respectively. These are typical values for such materials. All the bulk material properties are summarized in Table 3. It should be noted that the value of $\sigma_{0}$ for the adhesive is not representative of the yield stress of the material. It should be viewed as any other coefficient of the flow-law: its value is determined so as to give the best fit from a purely mathematical (i.e. a least-square regression analysis) point of view and it has no real physical meaning. The yield stress of the material which denotes the appearance of a significant amount of plastic deformation is best captured by the $0.2 \%$ yield limit which is equal to $36.7 \mathrm{MPa}$.

As noted above, the local fracture process is modelled employing a cohesive zone described by two 
parameters: the critical opening displacement $\delta_{c}$ and the peak stress $\hat{\sigma}$. Currently, there is no clear method for inferring these microscopic scale parameters directly from experimental results, although some researchers [19] have tried to address this issue. Hence, in the present work, these two parameters are determined by using an inverse analysis. Namely, their values are tuned to provide the best match with experimentally-measured, macroscopic, measurements obtained from a 'reference' test configuration. Three experimental values $\left(a, R_{1}\right.$ and $\left.R_{2}\right)$ obtained from wedge-peel tests, with $h_{a d h}=$ $0.25 \mathrm{~mm}$ and $h=1.45 \mathrm{~mm}$, were chosen as the reference test values to be used to ascertain these two parameters needed in the cohesive zone. (The wedge-peel test data are preferred to the fixed-arm peel test data to ascertain, and so fix, the values of these two parameters, since (i) an individual, single, test yields three $\left(a, R_{1}, R_{2}\right)$ instead of two $\left(P / b, R_{0}\right)$ experimental values, and (ii) because it is expected that the scatter is more likely to be reduced when using more numerous experimental-input values. Amongst the available wedge-peel test data, this particular specimen geometry was chosen (i) for its central position in terms of adhesive layer and adherend thickness, and (ii) because the corresponding experimental values show minimal scatter.) It should be noted that, by relying on only a single test configuration and a single specimen geometry to determine the values for these two parameters needed in the cohesive zone, it is intended to critically assess the predictions of the model when departing from these reference conditions, i.e. to examine the use of these values for the two parameters in modelling the other configurations of the wedge-peel test, as well as the fixed-arm peel tests.

From a numerical point of view, the two dimensional space of values (i.e. $\delta_{c}, \hat{\sigma}$ ) is coarsely spanned by letting each parameter vary in a range where the optimum is expected to be found. For each pair of values, the model of the wedge-peel test is used to predict the crack length and radii of curvature for the reference specimen geometry. The resulting discrepancy between numerical predictions and experimental values is evaluated quantitatively as an average, non-dimensional difference:

$$
\phi=\frac{1}{3}\left(\left|\frac{a^{\text {pred }}}{a^{\text {mes }}}-1\right|+\left|\frac{R_{1}^{\text {pred }}}{R_{1}^{\text {mes }}}-1\right|+\left|\frac{R_{2}^{\text {pred }}}{R_{2}^{\text {mes }}}-1\right|\right)
$$

where the superscripts mes and pred denote average experimental values and numerical predictions, respectively. Amongst all the pairs of parameters (i.e. $\delta_{c}, \hat{\sigma}$ ) envisaged, the one leading to the smallest discrepancy indicator is retained. The values of $\delta_{c}$ and $\hat{\sigma}$ are then perturbed and the model re-run iteratively until no further benefit can be achieved.

This optimization loop yielded the following values for the cohesive zone parameters:

$$
\delta_{c}=12.710^{-3} \mathrm{~mm} \quad \text { and } \quad \hat{\sigma}=99 \mathrm{MPa}
$$

which corresponds to an intrinsic work of fracture, $\Gamma_{0}$ :

$$
\Gamma_{0}=845 \mathrm{~J} / \mathrm{m}^{2} .
$$


When compared with the reference data, the numerical predictions all lie within the experimental scatter and show an average difference of $\pm 4.25 \%$. More details on the comparison between the experimental data and numerical predictions will be given in the next section.

\section{Numerical results}

\subsection{Wedge-peel test}

The model of the wedge-peel test was run for the three geometrical configurations which were tested experimentally. The corresponding results are presented, and compared with the experimental values, in Figures 8(a-c). Overall the agreement is very good, not only for the reference test (i.e. the wedgepee test with $h_{\text {adh }}=0.25 \mathrm{~mm}$ and $h=1.45 \mathrm{~mm}$ ) used for the identification of the two cohesive zone parameters $\hat{\sigma}$ and $\delta_{c}$, but also for the other specimen configurations. The largest discrepancy is found for the wedge-peel test where $h_{a d h}=0.40 \mathrm{~mm}$ and $h=1.45 \mathrm{~mm}$, and is of the order of $10 \%$ for the difference between the predicted and measured crack lengths. For this particular wedge-peel test configuration, the model does seem to underestimate slightly the energy required to fracture the adhesive, since the predicted values for both the radius of curvature on the crack side and the crack length are somewhat larger than the experimental values. (It is noteworthy that this wedge-peel test configuration is the only one associated with an adhesive layer thickness different from the reference test cases. Thus, the observed discrepancy may indicate that accounting in the model for an increase of the adhesive layer thickness by simply keeping the cohesive zone parameters fixed and placing solid elements throughout the adhesive layer might not be sufficient. Indeed, it could be argued that in the presence of a relatively thicker adhesive layer, the stress state in the fracture process zone, and hence both the damage mechanisms and the energy they dissipate, are modified. This would imply that the cohesive zone parameters $\left(\delta_{c}, \hat{\sigma}\right)$ might have to be modified accordingly for $\Gamma_{0}$.)

Values for the adhesive fracture energy, $G_{a}$, may also be derived from the modelling results. Following the partitioning made in the model, the value of $G_{a}$ is calculated as the sum of (i) the energy dissipated by the local damage mechanisms ahead of the crack tip, i.e. the intrinsic work of fracture $\Gamma_{0}$, and (ii) $\Gamma_{p}$, the plastic dissipation (plus any stored elastic strain energy) due to inelastic deformations within the adhesive layer but outside the fracture process zone, i.e. recall:

$$
G_{a}=\Gamma_{0}+\Gamma_{p} .
$$

The last term is integrated over the adhesive layer from the inlet section, where the strain energy is zero, down to the outlet section, where the material has completely unloaded:

$$
\Gamma_{p}=\int_{h_{\text {adh }}} \int_{X_{\text {in }}}^{X_{\text {out }}} S_{i j} \dot{E_{i j}} d X d Y
$$


which becomes, using Equation (6):

$$
\Gamma_{p}=-\int_{h_{\text {adh }}} \int_{X_{\text {in }}}^{X_{\text {out }}} S_{i j} \frac{\partial E_{i j}}{\partial X} d X d Y
$$

and is calculated numerically using the mid-point rule. It is worth noting that there is no contribution to $G_{a}$ coming from the energy dissipated, in bending for example, in the adherends.

Predicted values for the adhesive fracture energy, $G_{a}$, so deduced from the wedge-peel test are given in Figure 8(d). They reveal no significant dependence upon the thickness of either the adherend or the adhesive. This observation may be explained by the fact that the main contribution to $G_{a}$ comes from the local fracture process, i.e. $\Gamma_{0}$ which is the intrinsic work of fracture and is associated with the cohesive zone and which was assumed, in the model, to be a constant. (It should be noted that, for the present adhesive and loading configurations, a model based on a single row of cohesive elements for the entire adhesive layer, such as proposed by Yang and co-workers [9], might therefore be sufficient to properly reproduce the experimental results.)

For the sake of completeness, Figure 8(d) also includes values of $G_{a}$ obtained by Kawashita et al [20] from linear-elastic fracture-mechanics (LEFM) tests using a tapered-double cantilever-beam (TDCB) method. In these experiments the locus of joint failure was cohesive through the centre of the adhesive layer. Good agreement may be seen between the LEFM TDCB values for $G_{a}$ and the numericallydetermined wedge-peel values for $G_{a}$, especially for the specimens with $h_{a d h}=0.25 \mathrm{~mm}$. This observation further supports the idea that the value of the adhesive fracture energy, $G_{a}$, is independent upon the test configuration. However, the predicted value of $G_{a}$ from the wedge-peel test for $h_{a d h}=0.40$ $\mathrm{mm}$ appears to be somewhat lower than the LEFM value. This observation may arise for (i) the reasons discussed above in connection with the model for the wedge-peel test accounting for an increase of the adhesive layer thickness by simply keeping the cohesive zone parameters fixed and placing solid elements throughout the adhesive layer, or (ii) the observation that the locus of joint failure was close to an adhesive/adherend interface for the wedge-peel test but in the centre of the adhesive layer for the LEFM TDCB test.

Now, the plastic dissipation, $\Gamma_{p}$, in the adhesive layer appears, therefore, to only have a second-order effect on the value of the adhesive fracture energy, $G_{a}$. The evolution of $\Gamma_{p}$ as a function of the specimen geometry in the wedge-peel test is plotted in Figure 9. To help understand this evolution, the zones of active plasticity in the adhesive layer are plotted in Figure 10. They are defined as the regions where plastic dissipation occurs above the initial $0.2 \%$ yield strength of the material, which mathematically may be stated by:

$$
\left\{\begin{array}{c}
\dot{p}>0 \\
\bar{\sigma}>\sigma_{0}^{0.2 \%}
\end{array}\right.
$$


As shown in [11], the extent of the different plastic zones correlates with the amount of plastic dissipation, and their shape and location give an insight into the underlying mechanisms for their development. Firstly, considering Figure 10(b) which corresponds to $h_{\text {adh }}=0.25 \mathrm{~mm}$ and $h=1.45 \mathrm{~mm}$, two distinct zones can be identified [11]. Zone A extends from the crack tip over the complete thickness of the layer and arises from crack tip plasticity. Zone B is located behind the crack tip and runs along the extreme edge of the debonded adhesive layer. It can be attributed to bending of the arm and is prevented from occurring in the vicinity of the crack tip by reverse loading [21]. Secondly, considering Figure 10(a), which is for $h_{a d h}=0.25 \mathrm{~mm}$ and $h=1.00 \mathrm{~mm}$, an explanation can now be offered for the corresponding increase in $\Gamma_{p}$, see Figure 9: not only is the crack tip plasticity (Zone A) larger but the dissipation due to bending also increases, which is confirmed by the lower value of $R_{1}$. (It should be noted that Zone B in Figure 10(b) might appear to be larger in size compared with Figure 10(a), but it is important to realize that the plastic dissipation due to bending relates to the height of Zone $\mathrm{B}$ as the plasticity gets closer to the neutral axis; whereas its longitudinal extent follows the associated length scale in the problem, namely the crack length. Recalling that this latter term increases when changing the adherend thickness, $h$, from $1.00 \mathrm{~mm}$ to $1.45 \mathrm{~mm}$, it may thus be understood why Zone B extends further past the crack tip in Figure 10(b).) Thirdly, Figure 10(c) shows that much less plastic dissipation occurs in the adhesive layer of this wedge-peel test configuration, as reflected in the lower values of $\Gamma_{p}$, see Figure 9. Compared with Figure 10(b), Zone A shrinks towards the crack tip and does not now extend over the full thickness of the adhesive layer. Zone B is also smaller, since the radius of curvature is larger. Fourthly, it is worth mentioning that the dependence of $h_{a d h}$, and more particularly $h$, can partially be accounted for by subtracting the plastic dissipation in Zone B from $\Gamma_{p}$, on the grounds that this phenomenon is extrinsic to the fracture process, namely it arises from the bending of the arms. Finally, recent work by Martiny et al [11] has suggested a method for evaluating this extrinsic contribution and applied it with some success to the evaluation of $\Gamma_{p}$, and hence of $G_{a}$. (Interestingly, for the different adhesive then under study, the influence of this extrinsic contribution on the value of $\Gamma_{p}$, and hence value of $G_{a}$, was of far more significance than for the adhesive peel joints used in the present study.)

\subsection{Fixed-arm peel test}

The model of the fixed-arm peel test was run employing the specimen geometry, with $h_{\text {adh }}=0.40 \mathrm{~mm}$, and the peel angles used in the experiments, as described previously. The predicted peel force per unit width, $P / b$, is obtained directly from the present model. The maximum curvature, $1 / R_{0}$, of the peel arm is also obtained from the present numerical model, employing the smoothing and fitting methodology suggested in [13], based on the position, in the deformed geometry, of the nodes located at the edge of the peel arm.

The numerically-predicted results for the peel strength, $P / b$, and radius of curvature of the peel arm, $R_{0}$, are compared with their experimental counterparts in Figures 11(a-b), and the numerically-predicted values are generally lower than the experimental measurements. In terms of the peel force, this discrepancy is negligible for a peel angle of $135^{\circ}$ but increases with decreasing peel angles to reach a 
$12 \%$ difference at a $45^{\circ}$ peel angle; whereas the values of $R_{0}$ are all about $25-35 \%$ below the experimental values, regardless of the peel angle. These differences are considered to arise from an artifact caused by the assumption made in the modelling concerning the segment of the peel arm transmitting the force to the steady-state window of interest. (It will be recalled that it was assumed that this external arm was behaving elastically, while it was recognized it had actually undergone some plastic deformation. It is thus less compliant in the model than it should be and induces, for a given peel force, a larger bending moment at the crack tip. As a consequence, the predicted peel force and minimum radius of curvature of the peel arm are predicted to be relatively low in value). For increasing values of the peel angle, the bending moment-to-peel force ratio at steady-state crack growth increases. Thus, the differences between the experimental and numerically predicted values are decreased for the peel force, but more pronounced for the bending moment or, equivalently, the minimum radius of curvature analysis. These observations clearly demand an improvement in the way the boundary conditions are applied in the present model.

Now considering the adhesive fracture energy, $G_{a}$, it was derived numerically from Equation (12) by integrating from the inlet section, where the strain energy is zero, down to the outlet section where the peel arm shows zero curvature. These numerically-predicted $G_{a}$ values obtained from the present work are compared in Figure 11(c) with the corresponding values of $G_{a}$ which were calculated from the measured peel force per unit width, $P / b$, using the analytical model $[4,22]$. The agreement between the two approaches is encouraging. Especially, since it should be noted that the analytical model does make several approximations, but is relatively easy to apply in order to derive values of $G_{a}$. Indeed, it appears that the numerically-predicted values do give values of $G_{a}$ which are independent of the peel angle, whilst the analytically-derived values do show a small dependence of $G_{a}$ upon the peel angle. Figure 11(c) also includes, as horizontal lines, corresponding to $G_{a}$ values obtained experimentally from the LEFM TDCB (with $h_{a d h}=0.40 \mathrm{~mm}$ ) specimens and numerically from the model of the wedge-peel test (with $h_{a d h}=0.40 \mathrm{~mm}$ and $h=1.45 \mathrm{~mm}$ ), see Figure 8(d). Now, the numericallypredicted values of the $G_{a}$ from the fixed-arm peel test are also in good agreement with the wedge-peel test values shown earlier in Figure 8(d). However, the values of $G_{a}$ from the model proposed in the present work from both the wedge-peel and fixed-arm tests are somewhat lower than the values ascertained using the LEFM TDCB test specimen. It is difficult to state whether this is simply due to the predicted values from these two peel test configurations obviously being dependent upon the assumptions of the present model and/or from the accuracy of the fitted input data. Alternatively, the observation that the LEFM TDCB tests failed by crack growth through the centre of the adhesive layer, whereas the fixed-arm and wedge-peel tests failed by a cohesive fracture through the adhesive layer, but near one adhesive/adherend interface, might be of significance.

Notwithstanding, the accuracy of the present model applied to the problem of elastic-plastic peel test configurations should not be underestimated. The values of adhesive fracture energy, $G_{a}$, deduced from the numerical simulations proposed in the present paper, from all the various elastic-plastic peel test configurations, lie in the range of about $900 \pm 50 \mathrm{~J} / \mathrm{m}^{2}$; whilst the values from a previous analytical model [20] and a node-release finite-element analysis model [23], for a cohesive fracture of this adhesive, all lie in the range of about $1100 \pm 250 \mathrm{~J} / \mathrm{m}^{2}$. Thus, there is very good agreement between the 
different modelling methods. These values are clearly also in good agreement with the corresponding value from the well-established LEFM TDCB method of $1140 \pm 170 \mathrm{~J} / \mathrm{m}^{2}$, for $h_{a d h}=0.40 \mathrm{~mm}$.

As far as the plastic dissipation, $\Gamma_{p}$, in the adhesive layer is concerned, again it is found to have a second-order effect on the value of the adhesive fracture energy, $G_{a}$. The evolution of $\Gamma_{p}$ as a function of the peel angle in the fixed-arm peel test, for specimens with $h_{\text {adh }}=0.40 \mathrm{~mm}$ and $h=1.00 \mathrm{~mm}$, is plotted in Figure 12. The different values of $\Gamma_{p}$ are in excellent agreement, no matter the peel angle. Therefore, the value of the adhesive fracture energy, $G_{a}$, is, in the case of the fixed-arm peel test, independent of the peel angle. The only contribution to the overall energy expenditure that is significantly affected by the peel angle is the plastic dissipation in the flexible arm which, as reminder, is not included here in the calculation of $G_{a}$. These results are in agreement with the findings made, among others, by Guiu and Shanahan [24].

The zones of active plasticity in the $0.40 \mathrm{~mm}$ thick adhesive layer are plotted in Figure 13 for the fixedarm peel test for the three peel angles considered. These zones all appear to be very similar, as would be expected since the predicted $\Gamma_{p}$ values are not significantly different. Nevertheless, it should be noted that the assumption that Mode II (in-plane shear) does not play a significant role certainly reduces the possibility of any extra plastic dissipation which might be dependent on the peel angle. Further, comparing Figure 13 with Figure 10, where the latter figure shows the zones of active plasticity under wedge-peel test conditions, then two important effects may be observed. Firstly, Zone $B$ becomes non-existent in the fixed-arm peel test because the adhesive adheres to an infinitely rigid foundation that does not bend. Secondly, on the other hand, considering Zone A, the bending stresses occurring in the adhesive layer in wedge-peel test tend to push Zone A past the crack tip and so reduce its extent.

\section{Conclusions}

The present work has considered the numerical simulation of the steady-state fracture of adhesively bonded joints in various peel test configurations. The model is based on a multiscale approach involving the simulation of the continuum elastoplastic response of the adherends and the adhesive layer, as well as of the local fracture process taking place inside the adhesive layer using a cohesive zone formulation. A particular traction versus separation law was used in the cohesive zone formulation. It related the normal traction, $\sigma$, to the normal opening displacement, $\delta$, across the crack surface and showed no resistance in shear, since fracture was assumed to be predominantly via a Mode I (tensile) failure. The traction versus separation law was characterized by the peak stress, $\hat{\sigma}$, that can be sustained and the critical opening displacement, $\delta_{c}$, above which the normal tractions drop to zero. The area under the curve was denoted by $\Gamma_{0}$ and represented the intrinsic work of fracture, i.e. the energy dissipated per unit area of crack advance in the fracture process zone by the local damage mechanisms leading to fracture. It can therefore also be viewed as the value of the adhesive fracture energy, $G_{a}$, at crack initiation. The adhesive fracture energy, $G_{a}$, under steady-state conditions was evaluated as the sum of $\Gamma_{0}$ and of the plastic dissipation (plus any stored elastic energy), $\Gamma_{\mathrm{p}}$, in the adhesive as predicted by the continuum elastoplastic response of this latter. 
The fracture properties (i.e. $\hat{\sigma}$ and $\Gamma_{0}$ ) characterizing the adhesive were identified by an inverse analysis method based on knowing the residual radii of curvature and the crack length, both of these geometric terms being measured using an adhesively-bonded peel test specimen fractured using the wedge-peel test configuration. To validate the proposed numerical model, it was used, together with the now-fixed fracture properties, to predict the effect of various geometric features for other configurations of the wedge-peel test. The model was also applied to fixed-arm peel tests, subjected to various peel angles. The predicted results for the fixed-arm peel tests were also compared with experimentally measured peel forces and minimum radii of curvature.

All the numerical predictions relating to the wedge-peel test showed excellent agreement with the experimental data. Discrepancies between the numerical and experimental data were found to be more pronounced, though quite limited overall, for the fixed-arm peel tests. This was observed to be especially the case at low peel angles, leading in the worst case to a $37 \%$ difference in the predicted minimum radius of curvature, and a $12 \%$ difference in the predicted peel strength.

The numerical results from the proposed numerical model were also post-processed to extract values of the adhesive fracture energy, $G_{a}$, which was found to be not significantly dependent upon the details of the peel test configuration. This was attributed to the fact that the main contribution to $G_{a}$ arose from the intrinsic work of fracture, i.e. the energy dissipated locally ahead of the crack tip by the damage mechanisms leading to fracture. As a corollary, it was recognized that the far-field plastic dissipation occurring in the adhesive, but outside the fracture process zone, was a second-order effect for the specific joints and loading configurations analysed in this work.

The values of adhesive fracture energy, $G_{a}$, deduced from the numerical simulations proposed in the present paper, from all the various elastic-plastic peel test configurations, lie in the range of about 900 $\pm 50 \mathrm{~J} / \mathrm{m}^{2}$; whilst the values from a previous analytical model and a node-release finite-element analysis model, for a cohesive fracture of the present adhesive, all lie in the range of about $1100 \pm 250 \mathrm{~J} / \mathrm{m}^{2}$. Thus, there is very good agreement between the different modelling methods. These values are clearly also in good agreement with the corresponding value from the well-established LEFM TDCB method of $1140 \pm 170 \mathrm{~J} / \mathrm{m}^{2}$. 


\section{Acknowledgments}

The authors are grateful to Dr. L.F. Kawashita (Imperial College London), Dr. B.R. Blackman (Imperial College London) and M. M. Sinnaeve (UCL) for their participation in the experimental campaign on the wedge-peel test. Dr. L.F. Kawashita is also acknowledged for making his experimental data $[13,20]$ available to the present study, as well as Pr. C.M. Landis (Rice University) for providing the core of the finite element code on which our model was based. Finally, the present work was made possible thanks to Walloon Region (Belgium) and European ERDF funds obtained under contract number EP1A122030000102. 


\section{References}

[1] ASTM D 903. Standard test method for peel or stripping strength of adhesive bonds. ASTM International.

[2] ASTM D 1876. Standard test method for peel resistance of adhesives (T-peel test). ASTM International.

[3] ASTM D 3167. Standard test method for floating roller peel resistance of adhesives. ASTM International.

[4] Kinloch AJ, Lau CC, Williams JG. Int J Fracture 1994;66(1):45.

[5] Kawashita LF, Moore DR, Williams JG. J Adhesion 2004;80(3):147.

[6] Kim K-S, Aravas N. Int J Solids Struct 1988;24(4):417.

[7] Kawashita LF, Moore DR, Williams JG. J Adhesion 2005;81(6):561.

[8] Pardoen T, Ferracin T, Landis CM, Delannay F. J Mech Phys Solids 2005(9);53:1951.

[9] Yang QD, Thouless MD, Ward SM. J Mech Phys Solids 1999;47(6):1337.

[10] Ferracin T, Landis CM, Delannay F, Pardoen T. Int J Solids Struct 2003;40(11):2889.

[11] Martiny P, Kinloch AJ, Lani F, Landis CM, Pardoen T. In: Proc. $28^{\text {th }}$ Annual Meeting of the Adhesion Society. Mobile, USA, 2005. p.360.

[12] Dean GD, Duncan BC. Preparation and testing of bulk specimens of adhesives, NPL Measurement Good Practice Guide No 17, July 1998.

[13] Kawashita LF, Moore DR, Williams JG. J Mater Sci 2005;40(17):4541.

[14] Tvergaard V, Hutchinson JW. J Mech Phys Solids 1996;44(5):789.

[15]Cui J, Wang R, Sinclair AN, Spelt K. Int J Adhes Adhes 2003;23(3):199.

[16] Dean RH, Hutchinson JW. In: Fracture Mechanics, ASTM STP 700, 1980. p.384

[17]Landis CM, Pardoen T, Hutchinson JW. Mech Mater 2000;32(11):663.

[18] Swift HW. J Mech Phys Solids 1952;1(1):1.

[19] Ivankovic A, Pandya KC, Williams JG. Eng Fract Mech 2004;71(4-6):657.

[20]Kawashita LF, Kinloch AJ, Moore DR, Williams JG. Int J Adhes Adhes, submitted for publication.

[21]Drugan WJ, Rice JR, Sham T-L. J Mech Phys Solids 1982;30(6):447.

[22] Georgiou I, Hadavina H, Ivankovic A, Kinloch AJ, Tropsa V, Williams JG, J Adhesion 2003;79(3):239.

[23] Hadavinia H, Kawashita LF, Kinloch AJ, Moore DR, Williams JG. Eng Fract Mech 2006; 73(16): 2324.

[24] Guiu A, Shanahan ME. Int J Adhes Adhes 2002;22(5):415. 


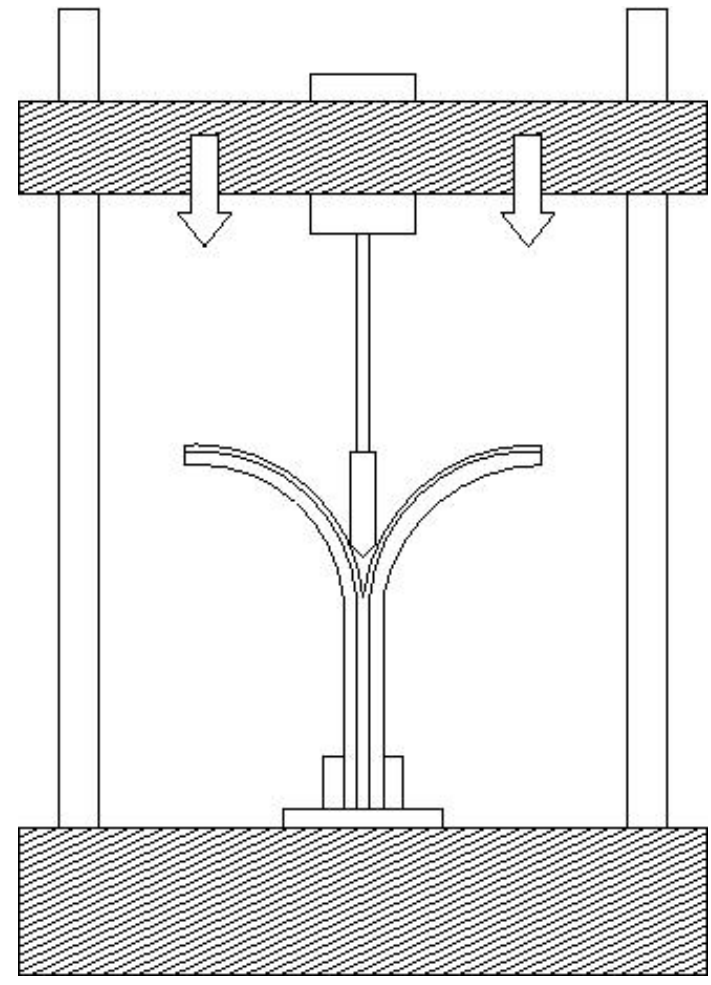

(a)

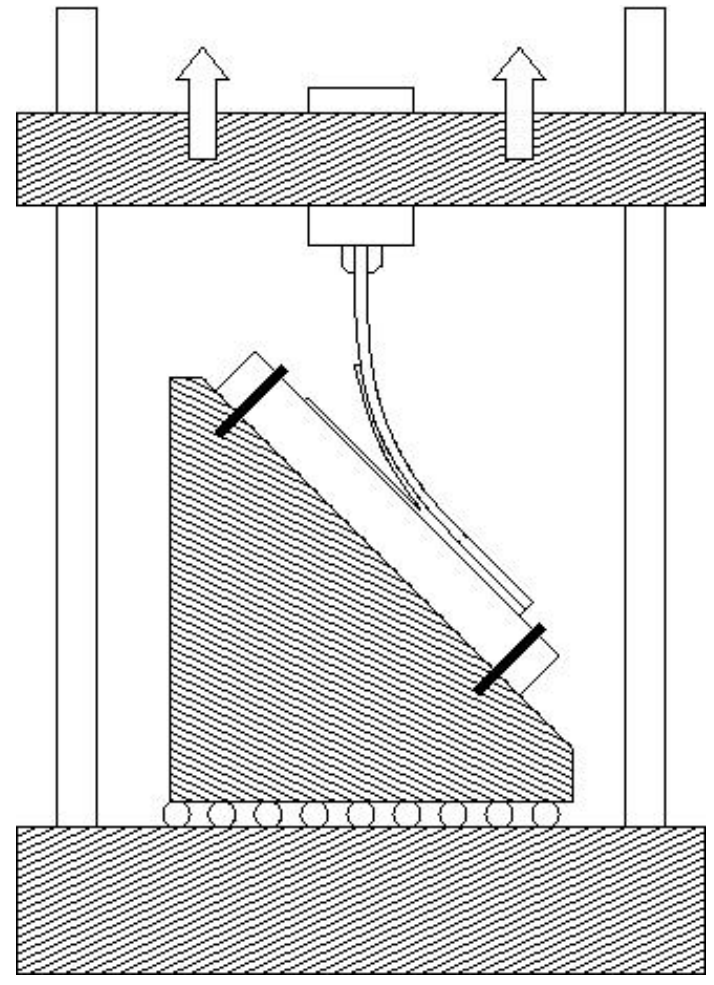

(b)

Figure 1: Experimental set-up for (a) the wedge-peel test and (b) the fixed-arm peel test 


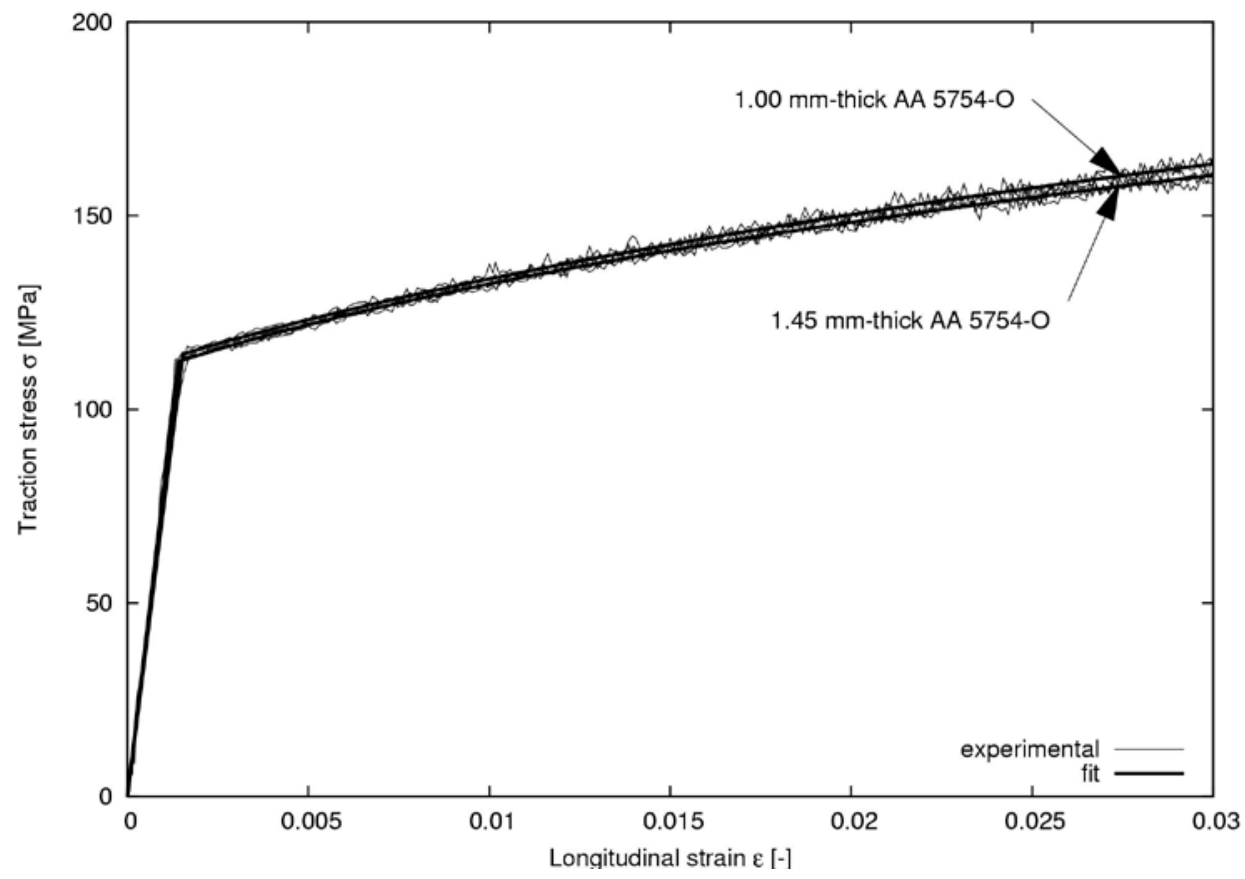

Figure 2: Experimental tensile stress versus strain curves and corresponding fits for AA 5754-O 


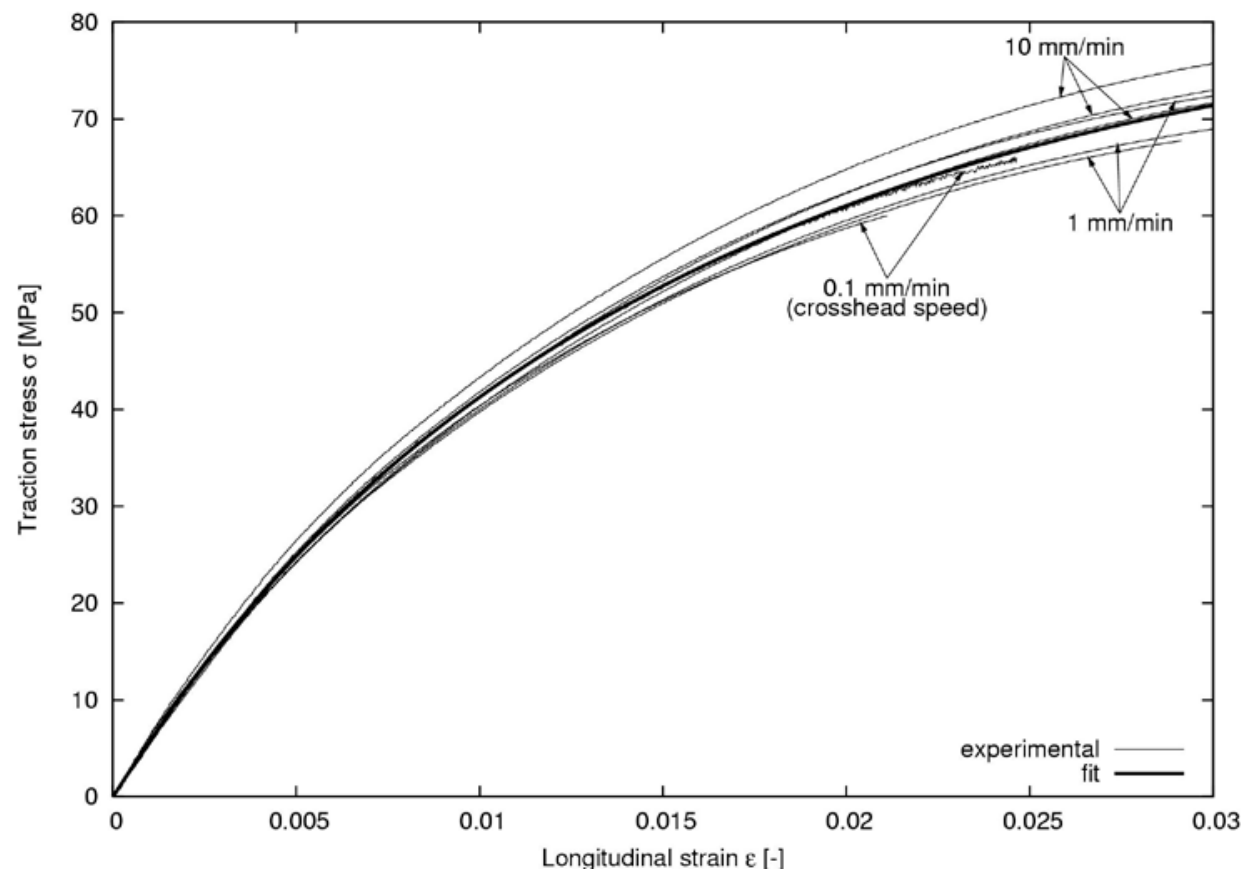

Figure 3: Experimental tensile stress versus strain curves and corresponding fit for adhesive ESP110 
adherend
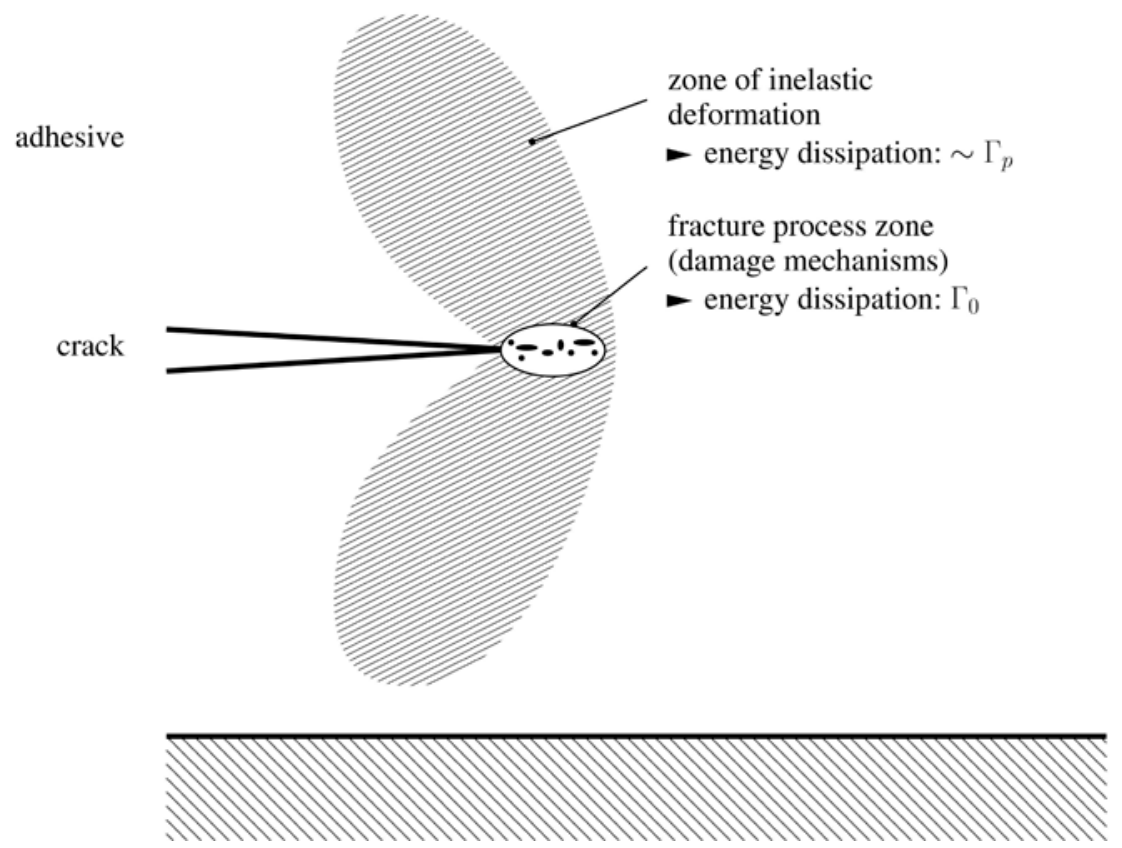

(a)

adherend

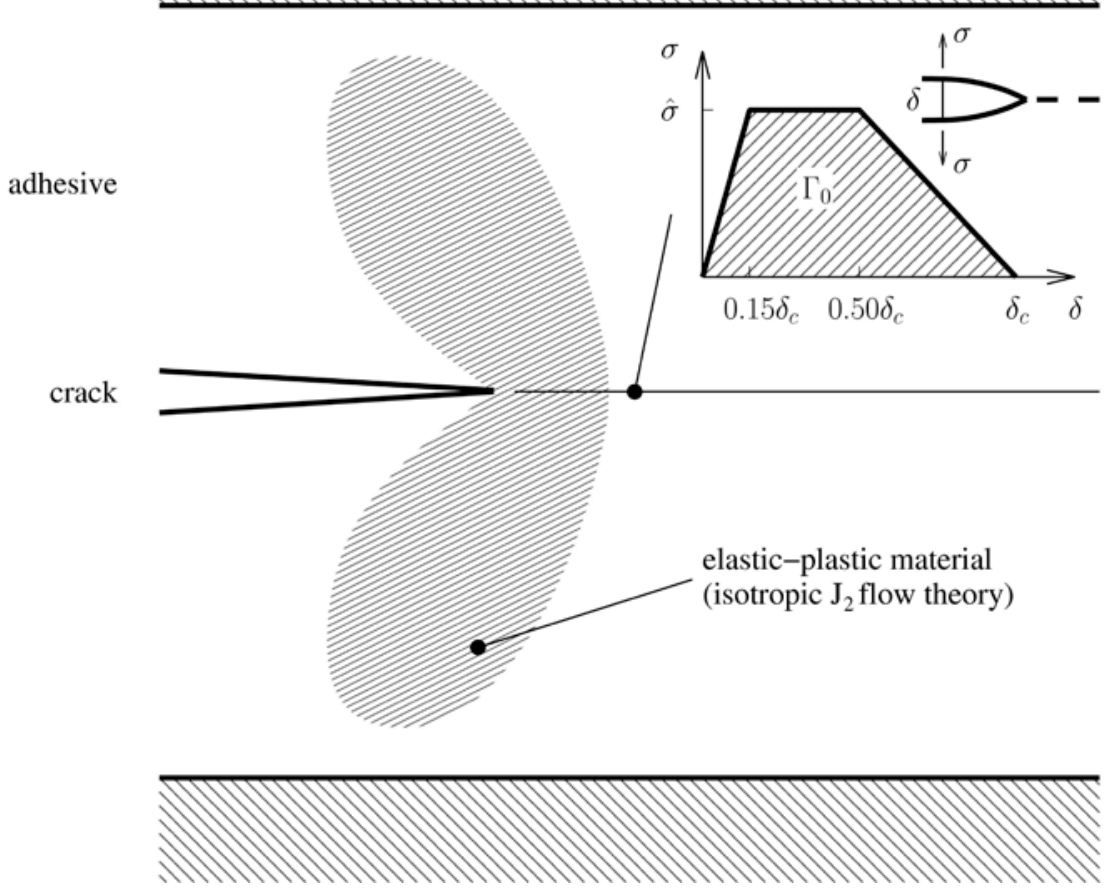

(b)

Figure 4: (a) Schematic representation of the physical phenomena occurring in the adhesive and (b) corresponding modelling in terms of a traction versus separation law 


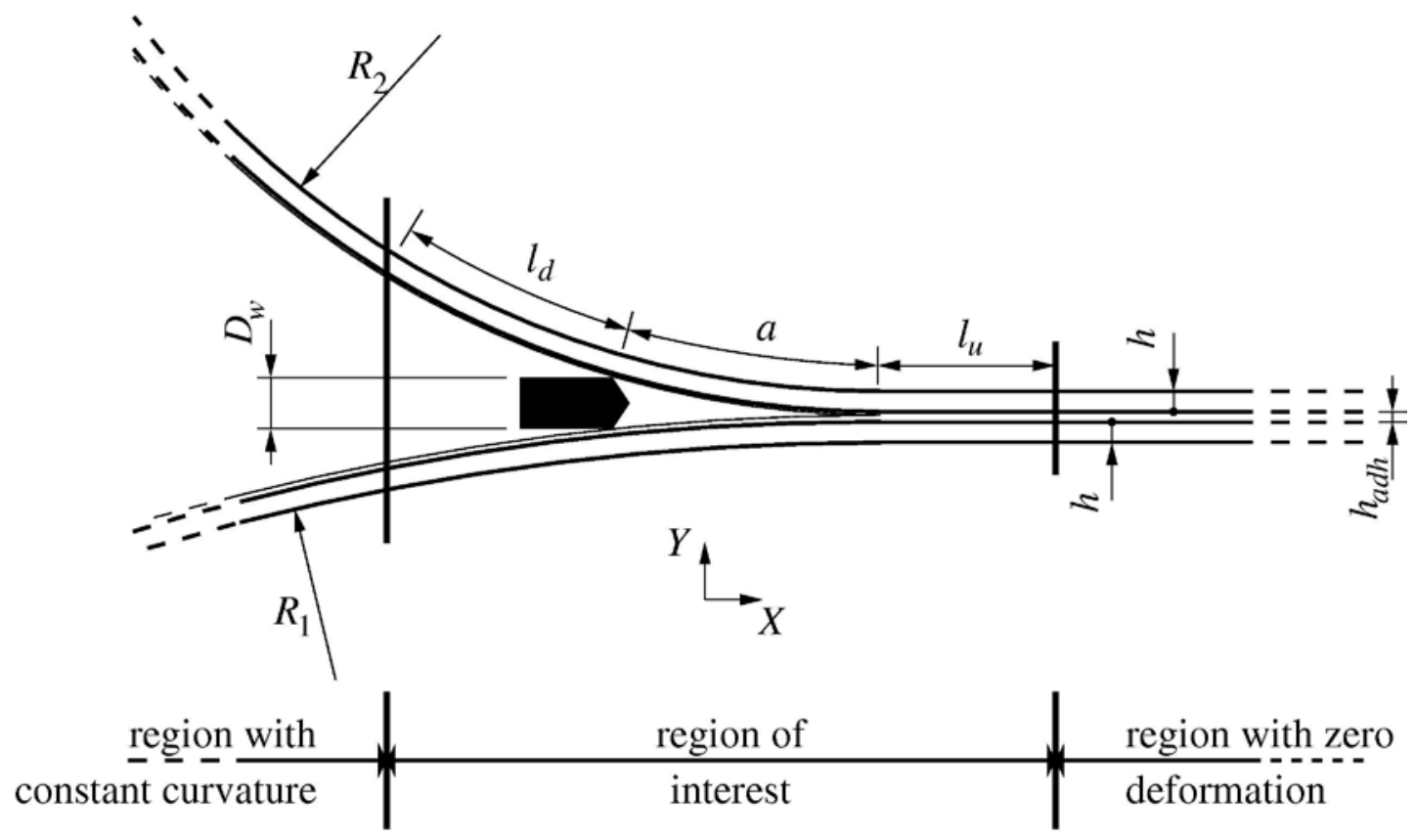

(a)

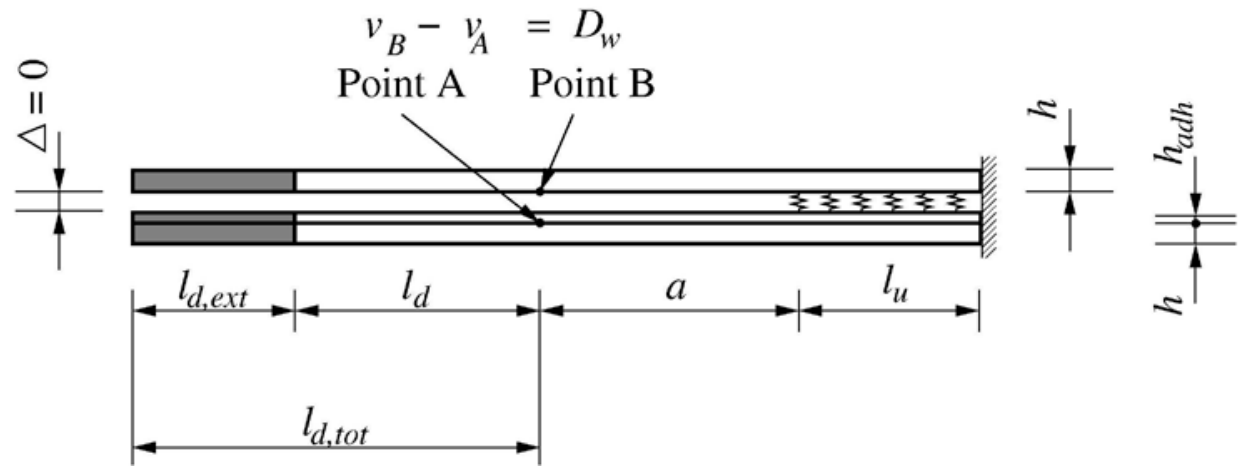

(b)

Figure 5: (a) Schematic representation of the wedge-peel test conditions and (b) corresponding boundary conditions used in the model 


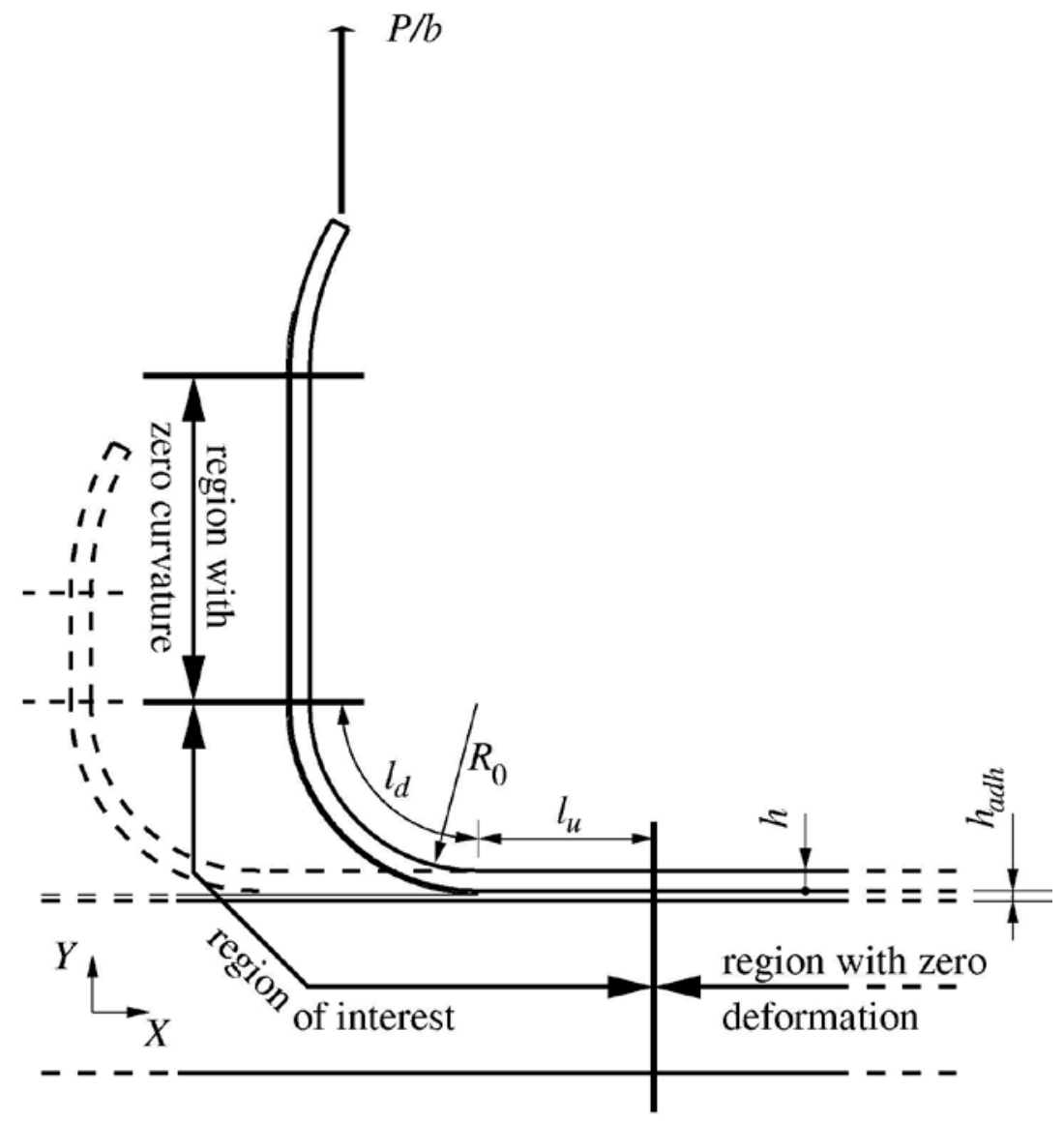

(a)
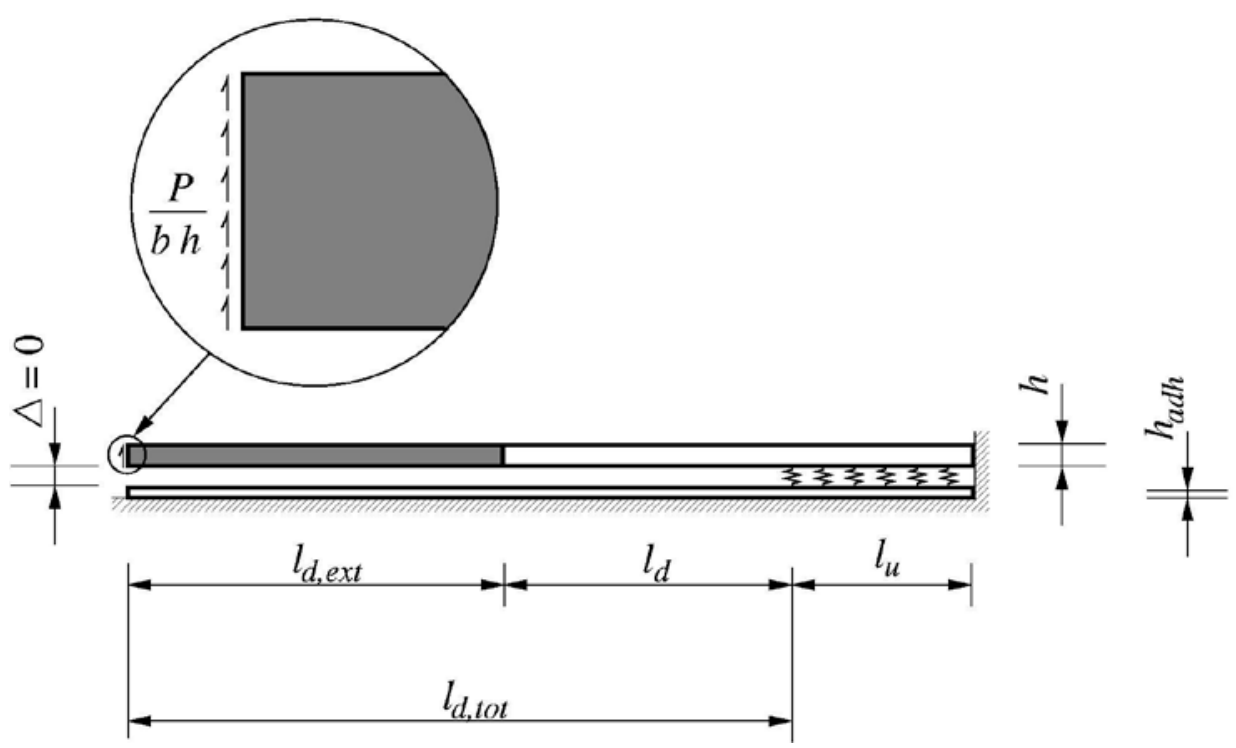

(b)

Figure 6: (a) Schematic representation of the fixed-arm peel test conditions and (b) corresponding boundary conditions used in the model 


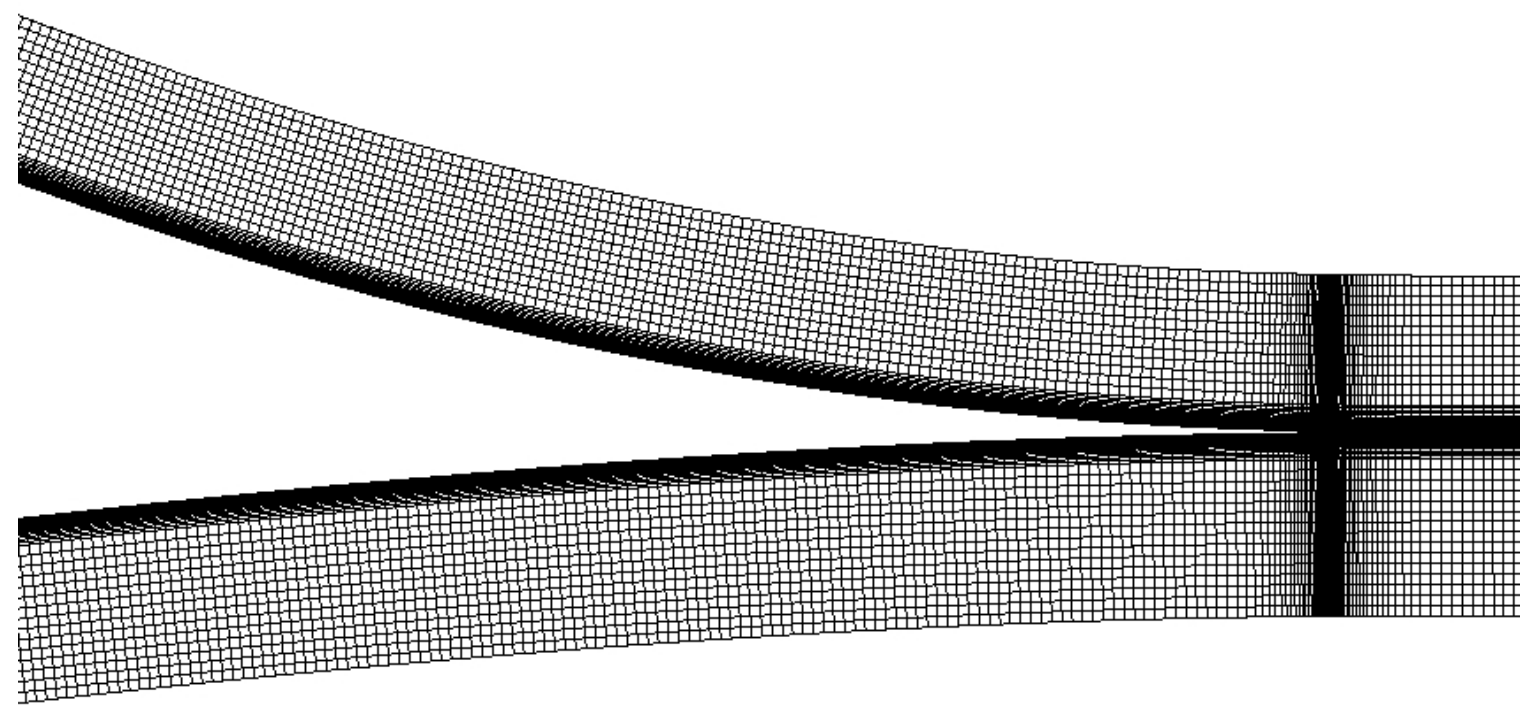

(a)

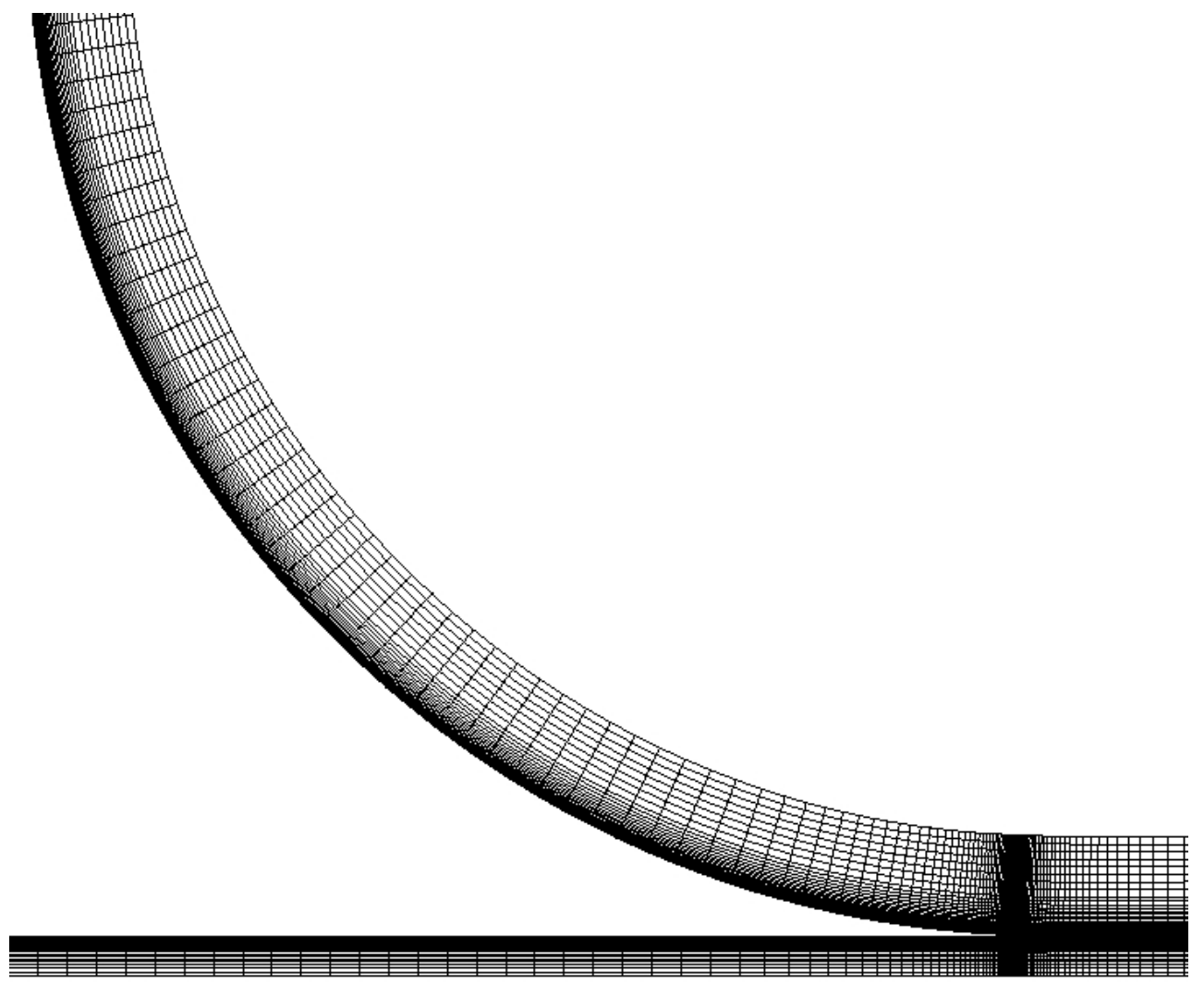

(b)

Figure 7: Typical meshes, in the deformed configuration, yielding accurate results when modelling (a) the wedge-peel test and (b) the fixed-arm peel test 


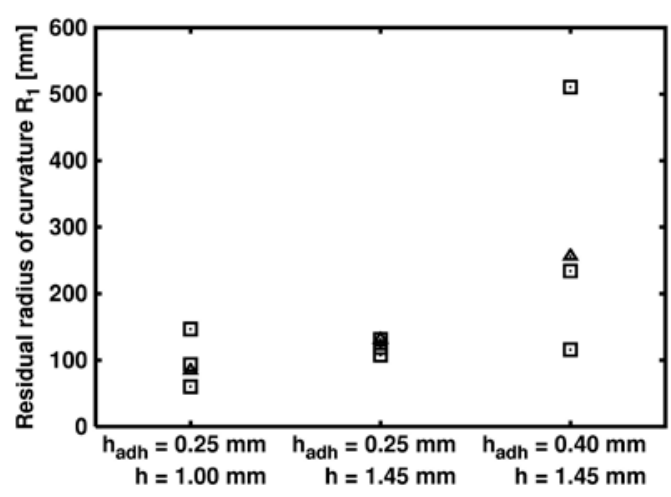

(a)

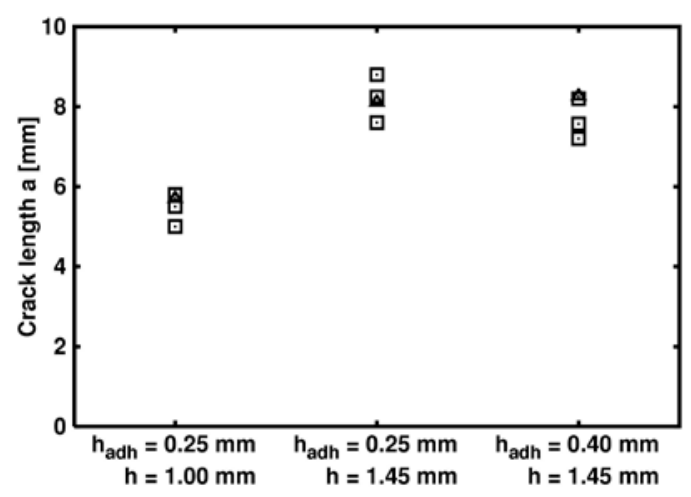

(c)

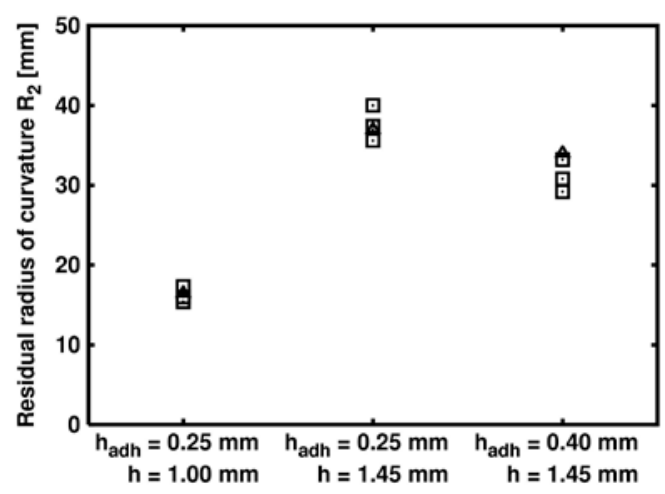

(b)

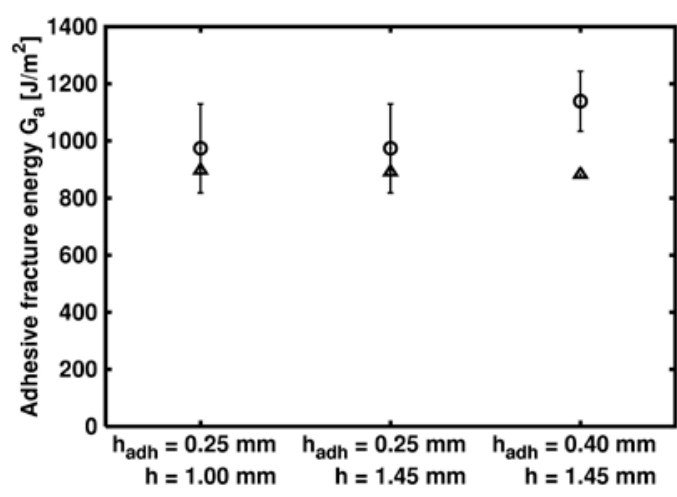

(d)

Figure 8: Comparison between experimental $(\square)$, if available, and numerical $(\triangle)$ values obtained for the wedge-peel test with $D_{w}=1.5 \mathrm{~mm}$ in terms of (a) the residual radius of curvature on the adhesive side, (b) the residual radius of curvature on the crack side, (c) the crack length and (d) the adhesive fracture energy. (In Figure8(d) the values of $G_{a}$ so determined are compared with the experimental

$$
\text { LEFM TDCB values (o).) }
$$




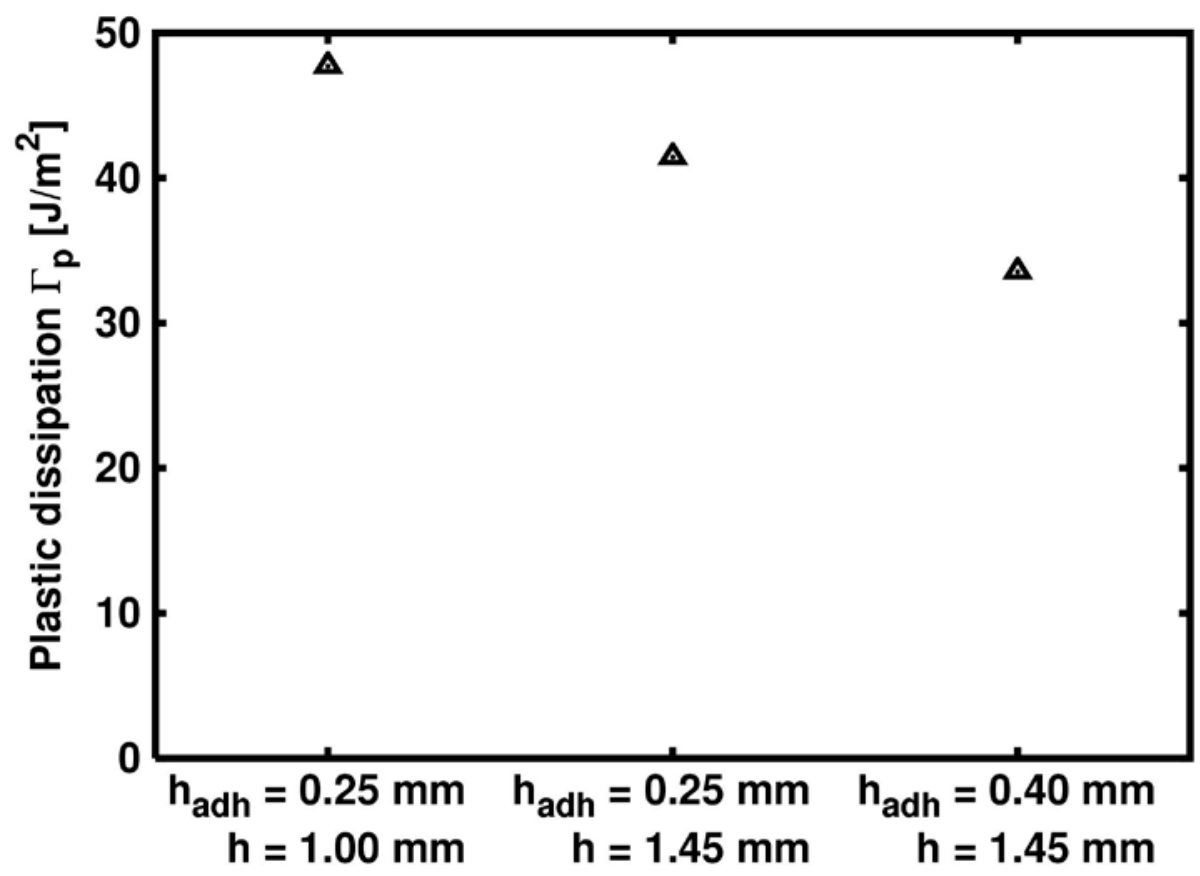

Figure 9: Plastic dissipation in the adhesive but outside the fracture process zone as predicted by the model of the wedge-peel test with $D_{w}=1.5 \mathrm{~mm}$ 


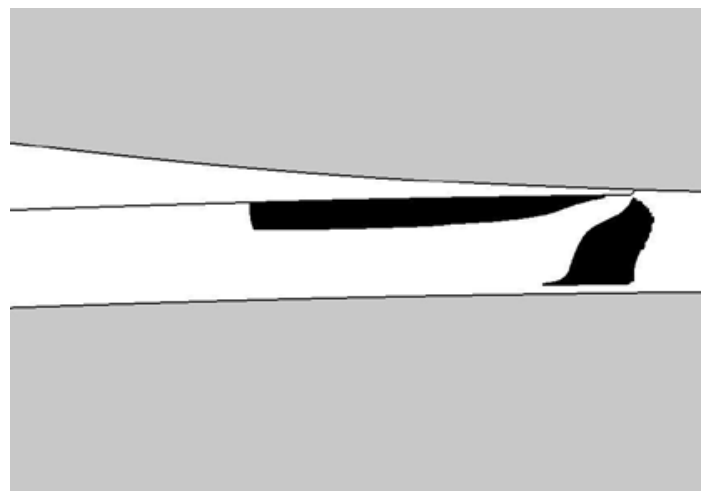

(a)

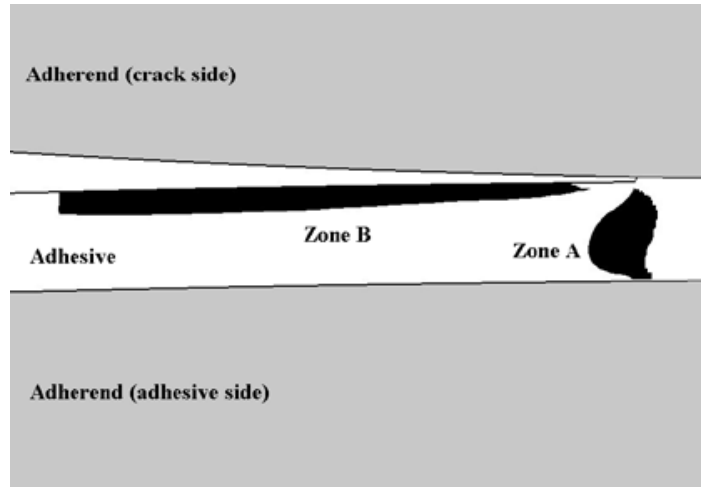

(b)
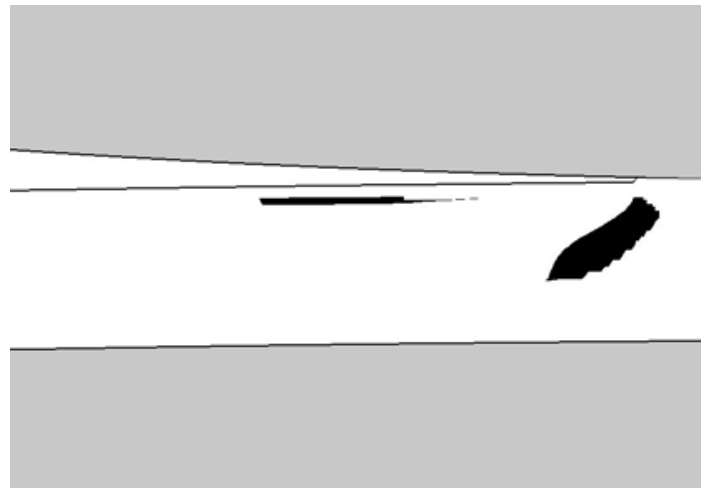

(c)

Figure 10: Zones of active plasticity in the adhesive obtained in the wedge-peel test with (a) $h_{\text {adh }}=0.25$ $\mathrm{mm}$ and $h=1.00 \mathrm{~mm}$, (b) $h_{\text {adh }}=0.25 \mathrm{~mm}$ and $h=1.45 \mathrm{~mm}$, and (c) $h_{\text {adh }}=0.40 \mathrm{~mm}$ and $h=1.45 \mathrm{~mm}$ 


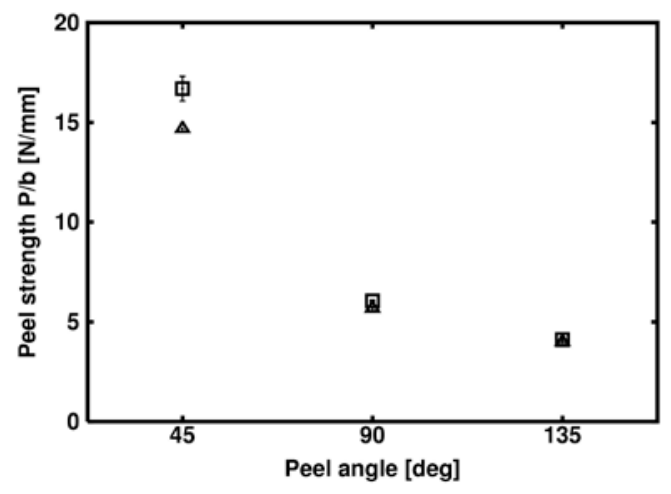

(a)

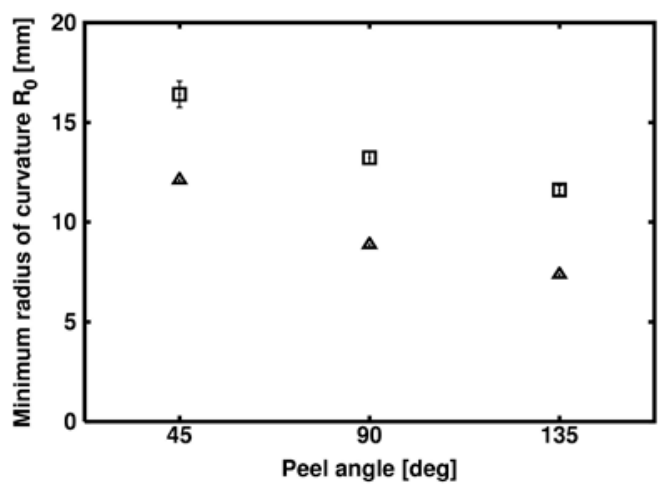

(b)

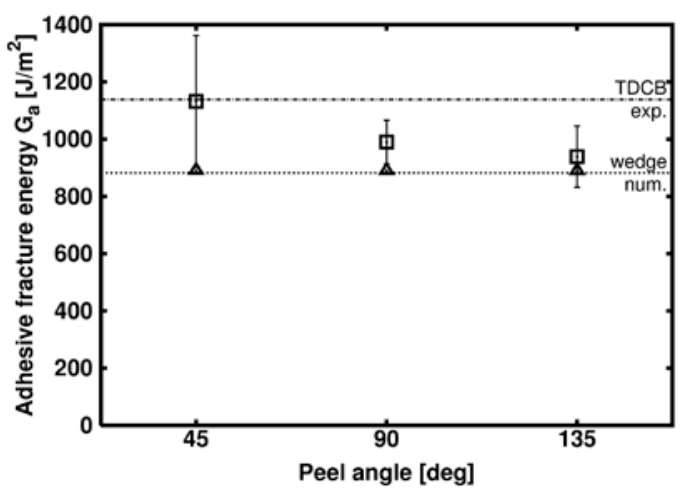

(c)

Figure 11: Comparison between experimental $(\square)$, or analytical in the case of

the adhesive fracture energy, $G_{a}$, and numerical $(\triangle)$ values obtained in the fixed-arm peel test, for specimens with $h_{a d h}=0.40 \mathrm{~mm}$ and $h=1.00 \mathrm{~mm}$, in terms of (a) the peel strength,(b) the minimum radius of curvature and (c) the adhesive fracture energy. (In Figure 11(c) the values of $G_{a}$ so determined are also compared with the corresponding numerical predictions (dotted line) obtained in the wedge-peel test and with the corresponding experimental LEFM TDCB results (dash-dotted line).) 


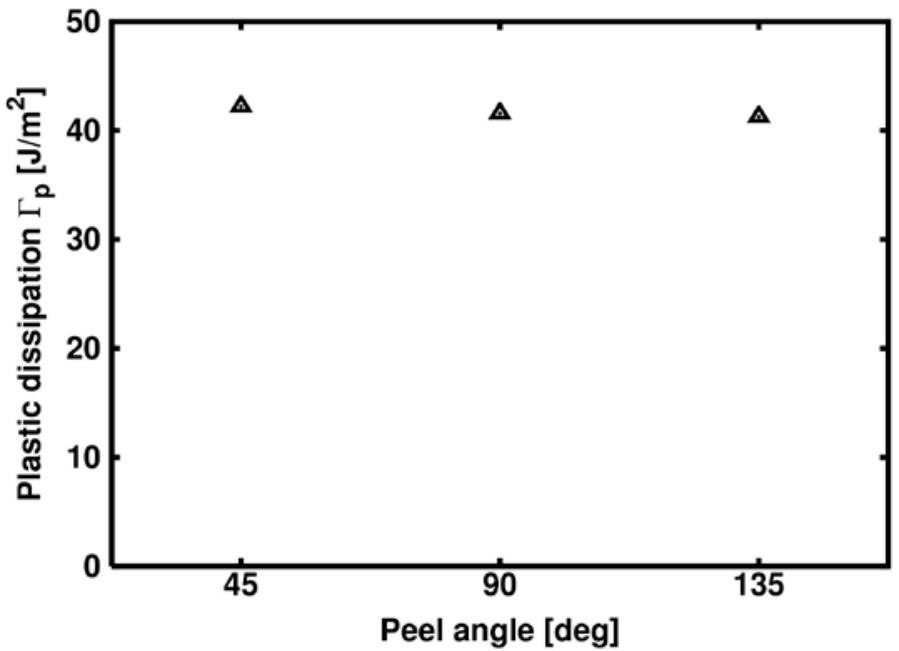

Figure 12: Plastic dissipation in the adhesive but outside the fracture process zone as predicted by the model of the fixed-arm peel test, for specimens with $h_{\text {adh }}=0.40 \mathrm{~mm}$ and $h=1.00 \mathrm{~mm}$ 


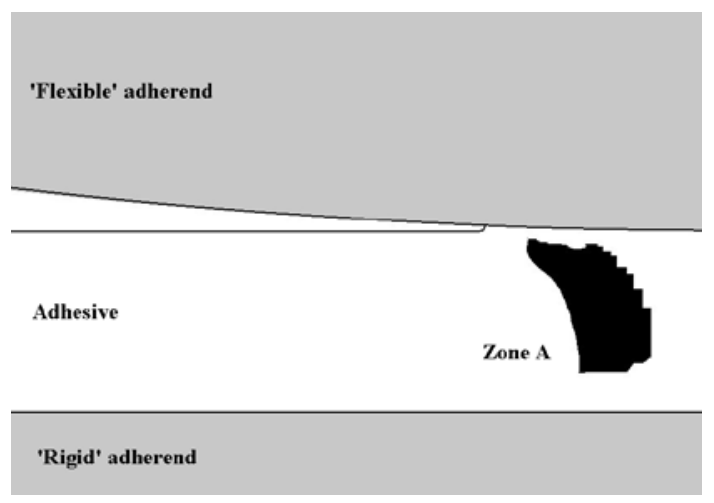

(a)

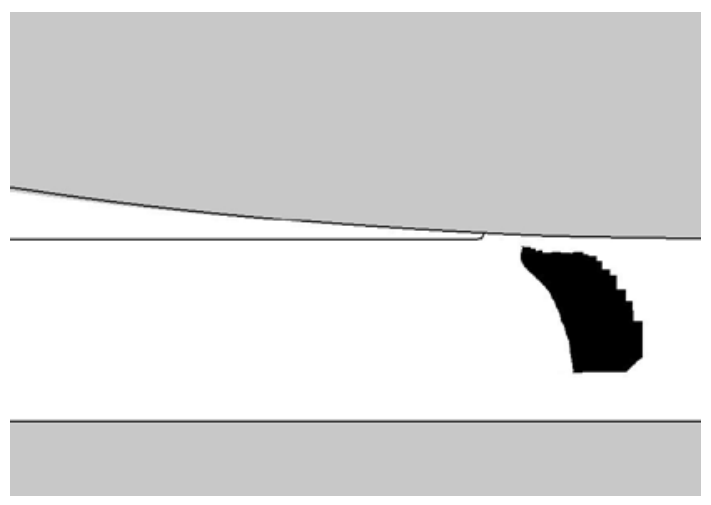

(b)

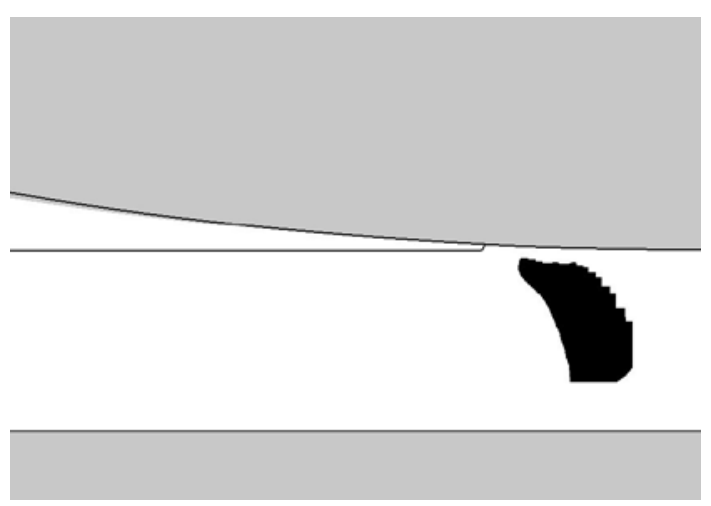

(c)

Figure 13: Zones of active plasticity in the adhesive obtained in the fixed-arm peel test with a peel angle of (a) 45 degrees, (b) 90 degrees and (c) 135 degrees 
Table 1: Experimental results obtained in the wedge-peel test with $D_{w}=1.5 \mathrm{~mm}$

\begin{tabular}{ccccc}
\hline $\boldsymbol{h}_{\boldsymbol{a d h}}[\mathbf{m m}]$ & $\boldsymbol{h}[\mathbf{m m}]$ & $\boldsymbol{a}[\mathbf{m m}]$ & $\boldsymbol{R}_{\mathbf{1}}[\mathbf{m m}]^{\mathrm{a}}$ & $\boldsymbol{R}_{\mathbf{2}}[\mathbf{m m}]^{\mathrm{a}}$ \\
\hline 0.25 & 1.00 & $5.4 \pm 0.4$ & $93.4 \begin{array}{l}+65.5 \\
-26.6\end{array}$ & $16.0 \begin{array}{l}+0.9 \\
-0.8\end{array}$ \\
\hline 0.25 & 1.45 & $8.2 \pm 0.6$ & $119 \begin{array}{l}+11 \\
-9\end{array}$ & $37.4 \begin{array}{l}+1.9 \\
-1.7\end{array}$ \\
\hline 0.40 & 1.45 & $7.7 \pm 0.5$ & $234 \begin{array}{l}+802 \\
-98\end{array}$ & $30.8 \begin{array}{l}+1.7 \\
-1.5\end{array}$ \\
\hline
\end{tabular}

${ }^{a}$ Average values and standard deviations are obtained in terms of the bending energy in the adherend. This energy is computed numerically with a commercial finite element code by considering the adherend without any adhesive, initially free of stress and subject to pure bending conditions. This explains why the average value is not located in the middle of the uncertainty interval.

Table 2: Experimental results obtained in the fixed-arm peel test on specimens with $h_{a d h}=0.40 \mathrm{~mm}$ and $h=1.00 \mathrm{~mm}$

\begin{tabular}{ccc}
\hline Peel angle [deg] & $\boldsymbol{P} / \boldsymbol{b}[\mathbf{N} / \mathbf{m m}]$ & $\boldsymbol{R}_{\mathbf{0}}[\mathbf{m m}]$ \\
\hline 45 & $16.7 \pm 0.6$ & $16.4 \pm 0.7$ \\
\hline 90 & $6.05 \pm 0.05$ & $13.2 \pm 0.29$ \\
\hline 135 & $4.11 \pm 0.12$ & $11.6 \pm 0.22$ \\
\hline
\end{tabular}

Table 3: Bulk material properties obtained by fitting the experimental stress versus strain curves

\begin{tabular}{lcccccc}
\hline Material & $\boldsymbol{E}[\mathrm{GPa}]$ & $\boldsymbol{v}[-]$ & $\sigma_{0}[\mathbf{M P a}]$ & $\boldsymbol{h}[\mathbf{M P a}]$ & $\boldsymbol{n}[-]$ & $\boldsymbol{k}[\mathbf{M P a}]$ \\
\hline AA 5754-O (1.00mm) & 74.7 & 0.33 & 114 & 87.5 & 0.290 & N/A \\
\hline AA 5754-O (1.45mm) & 79.5 & 0.33 & 112 & 107 & 0.257 & N/A \\
\hline ESP110 & 5.72 & 0.40 & 5 & 655 & 0.470 & -1800 \\
\hline
\end{tabular}

